\title{
Modelling imperfect advance demand information and analysis of optimal inventory policies
}

\author{
Tarkan Tan $^{\mathrm{a}, *}$, Refik Güllü ${ }^{\mathrm{b}}$, Nesim Erkip ${ }^{\mathrm{c}}$ \\ ${ }^{a}$ Department of Technology Management, Technische Universiteit Eindhoven, P.O. Box 513, 5600MB Eindhoven, The Netherlands \\ b Industrial Engineering Department, Bogazici University, 34342 Istanbul, Turkey \\ ${ }^{\mathrm{c}}$ Department of Industrial Engineering, Bilkent University, 06800 Ankara, Turkey
}

Received 15 May 2004; accepted 15 December 2005

Available online 28 February 2006

\begin{abstract}
We consider an inventory control problem where it is possible to collect some imperfect information on future demand. We refer to such information as imperfect Advance Demand Information (ADI), which may occur in different forms of applications. A simple example is a company that uses sales representatives to market its products, in which case the collection of sales representatives' information as to the number of customers interested in a product can generate an indication about the future sales of that product, hence it constitutes imperfect ADI. Other applications include internet retailing, Vendor Managed Inventory (VMI) applications and Collaborative Planning, Forecasting, and Replenishment (CPFR) environments. We develop a model that incorporates imperfect ADI with ordering decisions. Under our system settings, we show that the optimal policy is of order-up-to type, where the order level is a function of imperfect ADI. We also provide some characterizations of the optimal solution. We develop an expression for the expected cost benefits of imperfect ADI for the myopic problem. Our analytical and empirical findings reveal the conditions under which imperfect ADI is more valuable.
\end{abstract}

(C) 2006 Elsevier B.V. All rights reserved.

Keywords: Supply chain management; Inventory/production; Advance demand information; Dynamic base-stock policy; Periodic review

\section{Introduction and related literature}

There has been many improvements in Supply Chain Management (SCM) and inventory control, especially making use of developments in Information Technologies (IT) that made information flow faster, easier, and cheaper. Along with benefits such as decreasing demand variability by sharing information along supply chain members through means like Electronic Data Interchange (EDI), or decreasing lead times through means like faster and more accurate handling of demand information, there are also opportunities for further

\footnotetext{
* Corresponding author.

E-mail addresses: t.tan@tm.tue.nl (T. Tan), refik.gullu@boun.edu.tr (R. Güllü), nesim@bilkent.edu.tr (N. Erkip).
} 
improvements that make use of information. Such an opportunity may arise if information on future demand is employed, which is the subject of this study.

Information on future demand is referred to as Advance Demand Information (ADI), which is usually assumed to be perfect in the literature, that is customer orders that are available prior to their materialization are considered. In this study we focus on imperfect ADI, which means that early uncertain indication of prospective future orders is utilized.

The structure of imperfect ADI considered in this study covers a number of real life cases, some of which we discuss below. In most of these cases imperfect ADI already exists in the system of concern. As a consequence, it is easy and inexpensive to collect imperfect ADI in most applications.

Consider a company that uses sales representatives to market its products. The contact of a sales representative with a customer is prone to yield sales potential, unless the offer is rejected at once. In some cases the sales representatives prepare sales vouchers as means for giving quotations to the customers showing willingness to buy. Since it usually takes some time for a potential sale to materialize, the collection of sales representatives' information as to the number of customers interested in a product (such as the number of outstanding sales vouchers) can generate an indication about the future sales of that product, hence it constitutes imperfect ADI. In connection with this example, Easton and Moodie [6] discuss how "outstanding bids" (that is, pending proposals at prospective customers) can be employed in quoting the lead time and contract price for a new bid in a single resource production environment.

Internet retailing, by its nature, allows collection of imperfect ADI. A visit to a commercial web site is an indication of interest in one or more of the commodities (or services) offered by the company. Making use of links to more specific sub-pages or different forms of filtering are examples of tools that can be employed to differentiate between potential customers and the rest of the visitors. There are also other tools that can help to obtain more accurate ADI through the internet. Prospective (or actual) customers can fill in lists that clearly state the specific commodities they are interested in, or similarly they can prepare "wish lists" that can be used later for easier access to their preferred commodities when they have the necessary funding and/or time to realize the purchase. Alternatively, they can send the list to family and friends for birthdays or other special occasions, such as a wedding, to suggest gifts that can be purchased. Incomplete "shopping carts" also provide an indication on a customer's interests, since a customer with an incomplete shopping cart may finalize her order some other time. Another option is to add the possibility of watching the price changes for the commodities specified by the customer. The customer can be warned by e-mail (or by some other means such as mobile phone text message service) whenever there is a change in the price of a commodity she included in her list and/or whenever the price of the commodity drops below her preferred (and stated) price level. Some retailers do have such options in their web sites.

In business-to-business relations, retailers may share their forecasts with the supplier. Consequently, this information may serve as an action to reserve capacity, and hence the supplier can devise a probability structure to estimate their conversion into customer orders. A Vendor Managed Inventory (VMI) environment, in which the manufacturer is responsible for maintaining the supplier inventory levels, is a typical example. Also, as a complementing feature, consider "Collaborative Planning, Forecasting, and Replenishment" (CPFR), which is becoming more common (see CPFR site at www.cpfr.org). The CPFR Committee is a VICS (Voluntary Interindustry Commerce Standards) committee, made up of retailers, manufacturers, and solution providers, who developed a set of business processes that the entities taking place in a supply chain can use for collaboration. The mission of this committee is to create collaborative relationships between buyers and sellers through co-managed processes and shared information towards the aim of increasing the overall efficiency in the supply chain.

In this study we investigate the impact of using imperfect ADI on inventory policies. A decrease in uncertainty of future demand may allow the supplier to order in advance, which would shorten the duration between the placement of the actual demand by the customer and its delivery. Note that this time is shorter than the traditional lead time, as the order is placed before the actual demand occurs. The way we utilize imperfect ADI is through treating each individual ADI (e.g., each sales voucher) as a prospective demand, and assigning it a probability, $p$, of being realized as demand in the next time period. We note that the demand realization probability, $p$, may be referred to as "customer reliability level", as well. On the other hand, there is a probability, $r$, for which an ADI will remain in the system without being converted into a demand 
realization. We refer to $r$ as the "information sojourn rate". As we discuss in Section 2.1 this model structure enables us to represent reasonably complicated advance demand information environments. We consider periodic review ordering policies and we model the situation in the following manner:

- The total size of imperfect ADI, denoted by $k$, is the prospective number of demands available in a period (say yesterday), which includes both new customer information that has become available and those that had been collected previously and still remain in the system.

- A portion of the prospective demand materializes and becomes actual demand (each prospective demand with probability $p$ ) during the current period (say today).

- A portion of the prospective demand stays in the system for one or more number of periods (each prospective demand with probability $r$ ) before either becoming a demand or leaving the system.

- We assume that the materialized demand is the actual (realized) demand, (that is, there are no order cancellations) and is a function of $k$.

Note that there is a gap of at least one period between collecting ADI and receiving the actual demand. In other words, there is an imperfect information about a period's demand before its realization. This study intends to explore the impact of this information. We note that the number of customers that actually place orders may depend on ADI through a more complicated probability model. However, the simple multinomial model that we employ captures the partial realization of an ADI, and it can be estimated from customer demand history. In Section 4 we propose and analyze an extension to the unique customer reliability level in which the ADI is analyzed according to the sources that generate it and then segmented accordingly, each segment having its own customer reliability level.

When a demand has materialized, the customer order for the unit is due $l$ periods later. We refer to $l$ as "demand-lead-time" (as introduced by Hariharan and Zipkin [11]). While in some cases $l$ is zero, positive $l$ can be observed in many applications. This is especially common in service systems or customized products. Purchase agreements also constitute an example for a case of positive demand-lead-time. The time, $L$, that is required to satisfy an order (that is the traditional "lead time") will be referred to as "supply-lead-time". As we later demonstrate, the difference between $L$ and $l$ is what matters in determining inventory policies, rather than individual values of $L$ and $l$. The same result also holds in Hariharan and Zipkin [11] for a different model. They conclude that ADI improves system performance in the same way as a reduction in supplylead-times, under the situation of no order cancellation. Cheung and Zhang [3] model and analyze customer order cancellations, which they consider as an addition to the list of sources of "bullwhip effect". Bullwhip effect is a term introduced by Lee et al. [15], and it stands for the propagation of variance of demand along supply chain members. Most of the authors define ADI the way Hariharan and Zipkin do; that is, as perfect information on future demand. Our definition generalizes this concept to imperfect information, for which perfect information becomes a special case with $p=1$.

The literature on different forms of advance demand information has been rapidly increasing in recent years. Treharne and Sox [22] consider a non-stationary demand situation that can be partially observed, and hence produces partial information. Assuming that the demand in any given period arises from one of a finite collection of probability distributions, they model the demand as a composite-state, partially observed Markov decision process. Accordingly, they show that a state-dependent base stock policy is optimal for their problem environment. DeCroix and Mookerjee [4] consider a periodic-review problem in which there is an option of purchasing advance demand information at the beginning of each period. They consider two levels of demand information: Perfect information allows the decision maker to know the exact demand of the coming period, whereas the imperfect one identifies a particular posterior demand distribution. They characterize the optimal policy for the perfect information case. Gallego and Özer [7] model ADI through a vector of future demands and show the optimality of a state-dependent order-up-to policy. Van Donselaar et al. [23] investigate the effect of sharing uncertain ADI between the installers of a project and the manufacturers, in a project-based supply chain. The uncertainty in their setting arises from not having accomplished the selection of installers and manufacturers. Thonemann [21] elaborates further on a similar problem in which there is a single manufacturer and a number of installers. He considers two types of ADI: Information on whether or not the installers will place an order, and information on which product they will order. Zhu and Thonemann [25] consider a problem that 
consists of a number of customers that may provide their demand forecasts. These forecasts are employed to improve the demand forecast of the retailer through an additive Martingale model of forecast evolution. Assuming a linear cost associated with the number of customers that share information, they investigate the relation between the optimal number of customers to contact and the problem parameters.

Karaesmen et al. [14] consider a capacitated problem under ADI and stochastic lead times. They model the problem via a discrete time make-to-stock queue. Dellaert and Melo [5] model partial ADI in a make-to-stock environment through a Markov decision process given the existence of customer priorities and when the supplylead-time is negligible. ADI in this case is the currently committed demand on some constant number of periods in the future (with the exception of next period's demand information being perfect); that is, it is possible to receive more orders in those periods but not less, making the minimum demand known for these periods.

Our contributions in this study can be summarized as follows: (1) we present a fairly general probability structure for modelling imperfect advance demand information, (2) we demonstrate useful structural properties of the optimal policy, (3) under myopic policy we come up with an explicit expression for the expected cost benefits of employing imperfect advance demand information, (4) our computational results provide useful managerial insight for parameter settings where imperfect ADI becomes most beneficial.

Our probability model for representing the evolution of ADI records and the dynamic cost model are presented in Section 2. We characterize optimal policies in Section 3. We cover an extension of the problem in Section 4 where the ADI is segmented based on the sources that generate it. We investigate the value of ADI, first by elaborating on the myopic problem and then empirically solving the general problem in Section 5. We state our concluding remarks and possible extensions of this study in Section 6.

\section{Description of the model}

In this section our aim is twofold. We present our imperfect advance demand information model in Section 2.1. Then, in Section 2.2 we present a dynamic model that enables us to characterize optimal inventory policies under ADI and partial customer reliability. The notation is introduced as need arises, but we summarize our major notation in Table 1 for the ease of reference. Subscripts are omitted for simplicity, whenever unnecessary.

\subsection{Modelling imperfect advance demand information}

In our imperfect advance demand information model we let $M_{n}$ be the random variable denoting the size of advance demand information collected within period $n-1$ which becomes available at the beginning of period $n, n=1,2, \ldots$ We denote the observed realization of $M_{n}$ as $m_{n}$. We assume that $\left\{M_{n}, n=1,2, \ldots\right\}$ is an independent and identically distributed sequence with $\mu_{M}=E\left[M_{n}\right]$ and $\sigma_{M}^{2}=\operatorname{Var}\left[M_{n}\right]$. Customers who indicate their willingness to materialize their demands place their orders in period $n$, and the system observes the realized (actual) demand at the beginning of period $n+1$. Also let $K_{n}$ be the total number of prospective customers (total ADI size) who would be willing to place orders in periods $n, n+1, \ldots$ and $A_{n}$ be the number of customers who leave the system at the beginning of period $n$ without materializing any demand although they previously provided ADI. That is, $K_{n}$ is the number of potential customers who have been recorded as ADI in periods $t \leqslant n-1$, but have neither materialized their orders nor confirmed that they will not place any order, and $A_{n}$ is the number of customers who have been recorded as ADI until the beginning of period $n-1$ but declared during period $n-1$ that they decided not to place any order. Let $k_{n}$ be the realization of $K_{n}$. Note that $k_{n}$ also includes those customers whose advance demand information has just been collected $\left(m_{n}\right)$ in period $n-1$. Let $D_{n}(k)$ be the demand observed at the beginning of period $n$ (that is, collected in period $n-1$ ), as a function of total ADI size available at the beginning of period $n-1$. We assume that there are no other sources of demand, that is all of the demand is originated by the information generated in advance. We also assume that an arriving ADI in a period does not leave the system at the same period. Consequently, we can express the total ADI size available at the beginning of period $n+1$ as

$$
K_{n+1}\left(k_{n}\right)=k_{n}-D_{n+1}\left(k_{n}\right)-A_{n+1}\left(k_{n}\right)+M_{n+1} .
$$


Table 1

Relevant notation

\begin{tabular}{|c|c|}
\hline $\bar{N}$ & Number of decision epochs in the planning horizon \\
\hline$l$ & Demand-lead-time \\
\hline$L$ & Supply-lead-time \\
\hline$\tau$ & Effective lead time $(=L-l)$ \\
\hline$m_{n}$ & $\begin{array}{l}\text { Size of advance demand information which is accumulated within period } n-1 \text { and available } \\
\text { (observed) at the beginning of period } n\end{array}$ \\
\hline$M$ & Generic random variable denoting the size of an (unobserved) ADI which is accumulated in a period \\
\hline$\mu_{M}$ & Expected value of $M$ \\
\hline$\sigma_{M}^{2}$ & Variance of $M$ \\
\hline$k_{n}$ & Total size of advance demand information available at the beginning of period $n$ \\
\hline$K$ & Generic random variable denoting the total size of ADI available at the beginning of a period \\
\hline$D_{n+1}(k)$ & $\begin{array}{l}\text { Realized (actual) demand at the beginning of period } n+1 \text {, to be met at the beginning of period } n+l+1 \\
\text { (which is a function of the observed ADI, } k_{n}=k \text { ) }\end{array}$ \\
\hline$x_{n}^{c}$ & Net inventory carried during period $n$ \\
\hline$x_{n}$ & Effective inventory position \\
\hline$Q_{n}$ & Amount ordered at the beginning of period $n$ \\
\hline$y_{n}$ & Effective inventory position right after ordering $Q_{n}$ \\
\hline$f_{n}(x, k)$ & $\begin{array}{l}\text { Expected minimum cost of operating the system from the beginning of period } n \text { until the end of the } \\
\text { planning horizon when the effective inventory position at the beginning of period } n \text { is } x \text {, and } \\
\text { the size of available ADI on next period's demand is } k\end{array}$ \\
\hline$W_{n}(k)$ & $\begin{array}{l}\text { Random variable that denotes the demand that is realized during periods } n+1, n+2, \ldots, n+\tau \text {; that is, } \\
\text { during the effective lead time, given that } k \text { is the total size of ADI available in period } n\end{array}$ \\
\hline$G_{k}(w)$ & Distribution function of $W_{n}(k)$ \\
\hline$b$ & Penalty cost per unit of backorder per period \\
\hline$c$ & Production (or procurement) cost per unit \\
\hline$h$ & Inventory holding cost per unit per period \\
\hline$s$ & Salvage cost per unit (which is negative if salvage value exists) \\
\hline$p$ & Probability that an observed individual ADI record will be realized as demand \\
\hline$r$ & Probability that an observed individual ADI record waits in the system one more period \\
\hline$\alpha$ & Discounting factor $(0<\alpha \leqslant 1)$ \\
\hline
\end{tabular}

Each ADI record available at the beginning of period $n$ becomes a demand realization in period $n$ with probability $p>0$ or waits in the system for one additional period with probability $r \geqslant 0$, independent of how long it has been in the system, and leaves the system without becoming a demand realization with probability $q=1-p-r$. We assume that $p+r \leqslant 1$, and $r<1$. It directly follows that $A_{n}$ has Binomial distribution with parameters $k_{n-1}$ and $q$.

We can express $E\left[K_{n}\right]$ and $\operatorname{Var}\left[K_{n}\right]$ as follows:

$$
\begin{aligned}
& E\left[K_{n}\right]=\mu_{M} \sum_{i=0}^{n-1} r^{i}, \\
& \operatorname{Var}\left[K_{n}\right]=E\left[K_{n-1}\right] r(1-r)+\sigma_{M}^{2}+r^{2} \operatorname{Var}\left[K_{n-1}\right] .
\end{aligned}
$$

By taking the limits of these expressions as $n \rightarrow \infty$, we find out that at stationarity

$$
\begin{aligned}
& E[K]=\mu_{M} /(1-r), \\
& \operatorname{Var}[K]=\left(\mu_{M} r+\sigma_{M}^{2}\right) /\left(1-r^{2}\right) .
\end{aligned}
$$

The probability that an ADI record present in the system will ever become a demand realization in an infinite horizon is given by

$$
p^{E}=\sum_{i=1}^{\infty} p r^{i-1}=\frac{p}{1-r} \leqslant 1 .
$$


We note that (4) defines an upper bound in case of finite horizon, for which case the exact expression can be obtained by replacing the upper limit of the summation by the remaining number of periods to go. Similarly, $q^{E}:=1-p^{E}=q /(1-r)$ is the probability that an ADI record does not become a demand realization and eventually leaves the system. For the special case $r=0$, we have $q^{E}=q=1-p$, implying that each ADI record either becomes a demand realization in one period or leaves the system. In this case, total ADI size available at the beginning of period $n$ coincides with $m_{n}$ (as in this case no ADI record remains in the system for longer than one period), and hence $k_{n}=m_{n}$. Consequently, $K_{n}$ and $M_{n}$ are identical random variables when $r=0$.

We should note that the time each ADI record remains in the system until it becomes a demand realization (or before the end of horizon in the finite horizon case) is a defective geometric random variable (unless $p+r=1$ ), with $q^{E}$ being the probability that mass escapes infinity (indicating that an ADI record does not become demand realization). Using this observation one can devise a maximum likelihood estimation procedure for estimating $r$ and $p$ from the history of customer records.

In Section 2.2, when we demonstrate how ADI records can be utilized in determining optimal inventory policies, we will need the distribution of demand over a certain horizon of length $\tau \geqslant 1$. Let $k$ be the total size of ADI available at the beginning of period $n$, and $M_{n+1}, M_{n+2}, \ldots, M_{n+\tau-1}$ be random variables denoting advance demand information collected in periods $n, n+1, \ldots, n+\tau-2$, respectively. Let $W_{n}(k)$ be the random variable describing total demand over periods $n+1, n+2, \ldots, n+\tau$, for $\tau \geqslant 1$ :

$$
W_{n}(k)=\sum_{i=1}^{\tau} D_{n+i} .
$$

Since $D_{n+i}, i \geqslant 1$ depends on $k, W_{n}$ is also a function of $k$. Obviously, $D_{n+1}, D_{n+2}, \ldots, D_{n+\tau}$ are not independent random variables, unless $r=0$. Let $X_{1}$ be the random variable denoting the part of initial ADI size $k$ that becomes a demand realization in periods $n, n+1, \ldots, n+\tau-1$. Similarly, let $X_{i}$ be the part of $M_{n+i-1}$ that becomes a demand realization in periods $n+i-1, n+i, \ldots, n+\tau-1$, for $i=2,3, \ldots, \tau$. Notice that, by independence of $\left(k, M_{n+1}, M_{n+2}, \ldots, M_{n+\tau-1}\right),\left(X_{1}, X_{2}, \ldots, X_{\tau}\right)$ is an independent collection, and

$$
W_{n}(k)=\sum_{i=1}^{\tau} D_{n+i}=\sum_{i=1}^{\tau} X_{i} .
$$

It can easily be verified that $X_{1}$ has Binomial distribution with parameters $k$ and $p\left(1+r+r^{2}+\cdots+r^{\tau-1}\right)$. That is

$$
X_{1} \sim \operatorname{Binom}\left(k, p\left(1+r+r^{2}+\cdots+r^{\tau-1}\right)\right) .
$$

Similarly,

$$
X_{i} \mid M_{n+i-1} \sim \operatorname{Binom}\left(M_{n+i-1}, p\left(1+r+r^{2}+\cdots+r^{\tau-i}\right)\right)
$$

for $i=2,3, \ldots, \tau$. Therefore, conditioned on $M_{n+1}, \ldots, M_{n+\tau-1}, W_{n}(k)$ is distributed as sum of $\tau$ independent but non-identical Binomial random variables. As a consequence, $W_{n}(k)$ does not depend on $n$, hence we drop the subscript. Let

$$
u_{i}=p \sum_{j=0}^{\tau-i} r^{j}=p\left(1-r^{\tau-i+1}\right) /(1-r)
$$

for $i=1,2, \ldots, \tau$. Then, by conditioning $X_{i}$ on $M_{n+i-1}, i=2,3, \ldots, \tau$ we can show that

$$
\begin{aligned}
& E[W(k)]=k u_{1}+\mu_{M} \sum_{i=2}^{\tau} u_{i}, \\
& \operatorname{Var}[W(k)]=k u_{1}\left(1-u_{1}\right)+\sum_{i=2}^{\tau}\left\{\mu_{M} u_{i}\left(1-u_{i}\right)+u_{i}^{2} \sigma_{M}^{2}\right\} .
\end{aligned}
$$

We define $G_{k}(w)$ as the distribution function of $W(k)$,

$$
G_{k}(w)=\operatorname{Pr}\{W(k) \leqslant w\} .
$$


Evaluating the distribution of $W(k)$ is generally difficult. However, given the first two moments of the ADI generation model $\left(\mu_{M}\right.$ and $\left.\sigma_{M}^{2}\right)$ and customer reliability parameters ( $p$ and $r$ ) one can use Eqs. (5) and (6) to find the expected value and variance of $W(k)$.

For the important special case $r=0$ (this is a Bernoulli type imperfect ADI model, where each ADI either becomes a demand realization or leaves the system), we have $u_{i}=p$ for all $i=1,2, \ldots, \tau$ and

$$
\begin{aligned}
& E[W(k)]=k p+\mu_{M}(\tau-1) p, \\
& \operatorname{Var}[W(k)]=k p(1-p)+(\tau-1)\left\{\mu_{M} p(1-p)+p^{2} \sigma_{M}^{2}\right\} .
\end{aligned}
$$

\subsection{Development of the dynamic cost model}

In our dynamic model, the objective is to minimize the expected total discounted inventory-related costs. All unmet demand is backlogged. We assume linear holding, backorder, and unit production (or procurement) costs. We consider a finite horizon model, because it is more likely that the products to collect ADI are those with short life cycles. We also consider a discounting factor so that the time value of money can be regarded.

Let $N$ be the number of decision epochs in the planning horizon. Let $L$ and $l$ be the supply-lead-time and demand-lead-time, respectively. Consequently, we assume that the number of periods in the planning horizon is $N+L$ (the period at which the order placed in period $N$ is received). When the customer demand is realized, the system commits itself to satisfy the demand after $l$ periods. Let $Q_{n}$ be the quantity ordered at the beginning of period $n$, and let $x_{n}^{c}$ be the net inventory carried during period $n$. The problem can be illustrated as in Fig. 1 for the whole planning horizon, and as in Fig. 2 for a specific period $n$.

For each period $n$ the following order of events take place:

- At the beginning of period $n, Q_{n-L}$ arrives.

- $D_{n-l}$ is met/backordered.

- $D_{n}$ is realized.

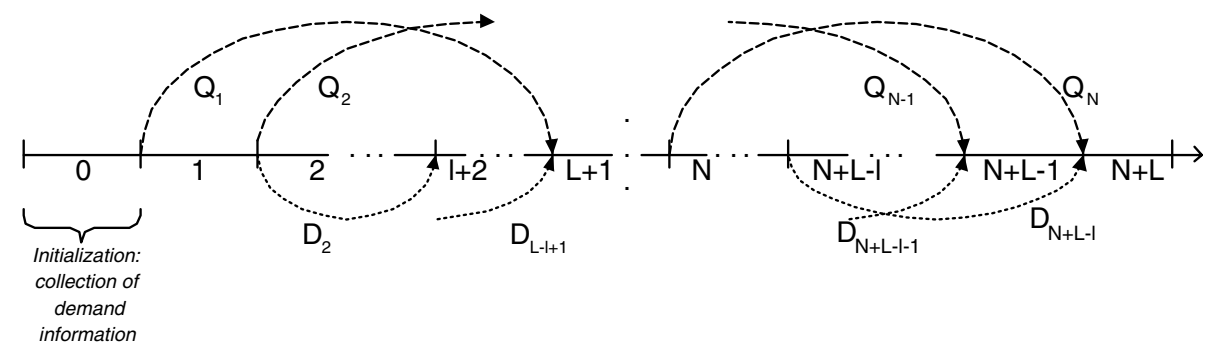

Fig. 1. Finite horizon problem.

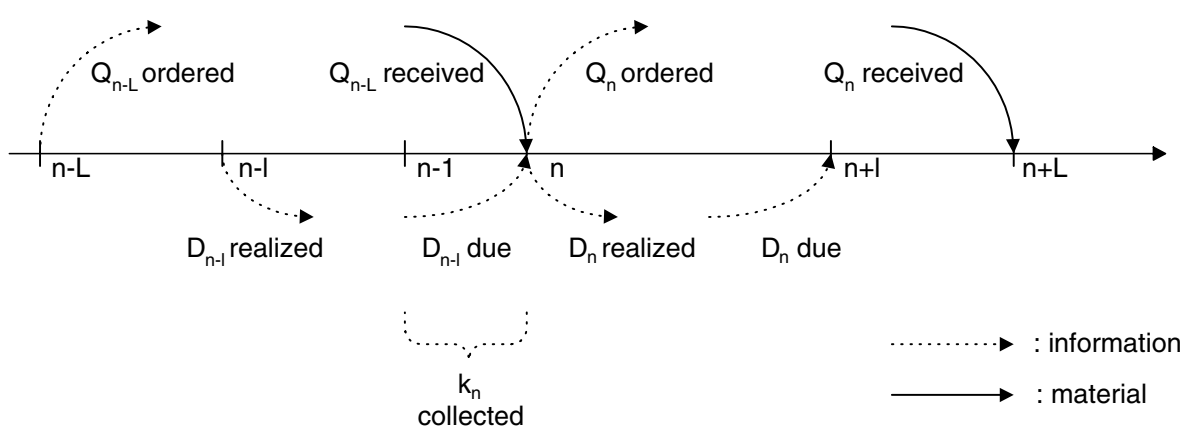

Fig. 2. Illustration for period $n$. 
- $x_{n}^{c}$ is updated.

- $m_{n}$ is observed.

- $k_{n}$ is updated.

- $Q_{n}$ is ordered.

We first note that for $L \leqslant l$ the problem is trivial, as the value of imperfect advance demand information is zero; because, the system can always match demand by appropriately adjusting the times of orders. Hence we consider the more interesting case of $L>l$.

The first demand is assumed to be realized at the beginning of period 2. This demand is the collection of the individual demands that occurred during period 1 for which advance demand information is collected in the "initialization" phase (period 0). The last demand is assumed to be realized at the beginning of period $N+L-l$ which is intended to be received at the beginning of period $N+L$, that is, the end of the planning horizon.

Since at the beginning of period $n$, right after ordering $Q_{n}$, nothing can be done to influence the net inventory until period $n+L$, inventory related costs associated with period $n+L$ can be accounted in period $n$ as

$$
c Q_{n}+\alpha^{L}\left(h E\left[x_{n+L}^{c}\right]^{+}+b E\left[x_{n+L}^{c}\right]^{-}\right),
$$

where $c$ is the unit production (or procurement) cost, $h$ and $b$ are the per period holding and backorder costs, respectively.

The usual net-inventory recursion can be noted as

$$
x_{n+1}^{c}=x_{n}^{c}+Q_{n+1-L}-D_{n+1-l} .
$$

Successive substitution in (10) results in

$$
x_{n}^{c}=\sum_{i=1}^{n-L} Q_{i}-\sum_{i=2}^{n-l} D_{i}
$$

for $n \geqslant L+1$, assuming that $x_{1}^{c}$ is zero, without loss of generality. We do not consider the costs that may be incurred before period $L+1$, as the first ordering possibility arises at the beginning of period 1 (which is received at the beginning of period $L+1$ ), and therefore there is no way to influence the costs in periods $1,2, \ldots, L$. We also note that by using (11)

$$
x_{n+L}^{c}=x_{n}^{c}+\sum_{i=n-L+1}^{n} Q_{i}-\sum_{i=n-l+1}^{n+L-l} D_{i} .
$$

Rearranging the terms, Eq. (12) can also be expressed as

$$
x_{n+L}^{c}=\left(x_{n}^{c}+\sum_{i=n-L+1}^{n-1} Q_{i}-\sum_{i=n-l+1}^{n} D_{i}\right)+Q_{n}-\sum_{i=n+1}^{n+L-l} D_{i} .
$$

The term in parenthesis in (13) is the traditional inventory position definition for period $n$ right before ordering, with the difference that the demand that has been realized as of period $n$ but not due yet is subtracted from it. We refer to this term as "effective inventory position" and denote it as $x_{n}$; that is,

$$
x_{n}=x_{n}^{c}+\sum_{i=n-L+1}^{n-1} Q_{i}-\sum_{i=n-l+1}^{n} D_{i} .
$$

The rightmost summation in (13) is the total demand that will be realized between periods $n+1$ and $n+L-l$. This term is the demand during "effective lead time", where effective lead time, $\tau$, is defined as the difference between the supply-lead-time and the demand-lead-time:

$$
\tau=L-l \text {. }
$$

Hence, (13) can be re-stated as

$$
x_{n+L}^{c}=x_{n}+Q_{n}-\sum_{i=1}^{\tau} D_{n+i} .
$$


Let us denote the random variable that describes the total demand during effective lead time as $W(k)$, as introduced in Section 2.1, that is,

$$
W(k)=\sum_{i=1}^{\tau} D_{n+i} .
$$

Finally, $x_{n}+Q_{n}$ can be viewed as the level that the system raises the effective inventory position up-to. Let us denote this as $y_{n}$, that is, $y_{n}=x_{n}+Q_{n}$. Consequently,

$$
x_{n+L}^{c}=y_{n}-W(k) .
$$

At the beginning of period $n$, the system state that is available to decide on $Q_{n}$ is made up of $x$ (effective inventory position at the beginning of period $n$ ), and $k$ (the size of available ADI). We define $f_{n}(x, k)$ as the expected minimum cost of operating the system from the beginning of period $n$ until the end of the planning horizon; that is,

$$
f_{n}(x, k)=-c x+\min _{y \geqslant x}\left\{J_{n}(y, k)\right\},
$$

where

$$
J_{n}(y, k)=\mathscr{L}(y, k)+\alpha E\left[f_{n+1}\left(y-D(k), K_{n+1}(k)\right)\right]
$$

for $1 \leqslant n \leqslant N$,

$$
\mathscr{L}(y, k)=c y+\alpha^{L}\left(h \int_{0}^{y}(y-w) \mathrm{d} G_{k}(w)+b \int_{y}^{\infty}(w-y) \mathrm{d} G_{k}(w)\right),
$$

and $K_{n+1}(k)=k-D(k)-A(k)+M$, as expressed in Eq. (1). We assume that the remaining inventory can be salvaged with a unit revenue of $c$ and outstanding backorders are satisfied with a unit cost of $c$ at the end of the planning horizon, that is, $f_{N+1}(x, k)=-c x$.

\section{Characterization of the optimal policy}

In this section, we obtain structural results about the finite horizon model introduced in Section 2 and its optimal solution. As we demonstrated in Section 2, at least one ingredient of $W(k), X_{1}$, is a discrete random variable. Therefore, $G_{k}(w)$ is not continuous. However, for the ease of exposition we assume $G_{k}(w)$ is continuous and $\mathscr{L}(y, k)$ is twice differentiable. Our results also hold for the discrete case. We first note that $\mathscr{L}(y, k)$ is convex in $y$ for all $k \geqslant 0$, since it is the usual newsboy cost function.

Theorem 1. The following properties hold for $n=1,2, \ldots, N$.

(i) $J_{n}(y, k)$ is convex in $y$, for all $k \geqslant 0$.

(ii) $f_{n}(x, k)$ is convex in $x$, for all $k \geqslant 0$.

(iii) Let $y_{n}(k)$ be the value of $y$ that minimizes $J_{n}(y, k)$. Then, the optimal ordering policy at the beginning of period $n$ is of state-dependent order-up-to type which is defined by

$$
Q_{n}= \begin{cases}y_{n}(k)-x, & \text { if } x<y_{n}(k), \\ 0, & \text { if } x \geqslant y_{n}(k) .\end{cases}
$$

Proof. Proof is provided in Appendix A.

Theorem 1 reveals that, upon observing the system state $(x, k)$ at the beginning of period $n$, the optimal policy is to order an amount that will bring the effective inventory position of the system to $y_{n}(k)$. Sethi and Cheng [18] (also Song and Zipkin [19], and Chen and Song [2] for other similar cases) have shown the optimality of statedependent order-up-to type policies (or state-dependent $(s, S)$ type policies under fixed ordering costs) when there exists a Markov-modulated demand process. In our case we have a simple and more explicit structure, and it is not straightforward to show that ours is a special case of the general problem structure. Our Theorem 1 is in line with Remark 4.5 of Sethi and Cheng [18], which claims that their optimality result can be extended to the case 
where there are countably infinite states describing demand. We note that the demand process in our problem can be pictured as a Markov-modulated process with countably infinite states describing demand/information structure, because $M_{n}$ is defined as an independent and identically distributed (iid) sequence. This allows construction of a stationary probability transition matrix between information states (ADI sizes) that describe demand during effective lead time. Nevertheless, Theorem 1 can also be extended to the case where $M_{n}$ is not iid, that is when the Markovian structure does not hold. This requires redefining the stationary and independent elements in our model that depend on the total ADI size only, but the general line of the proof remains the same. In that case total available ADI size would not be enough for describing demand during lead time, because of the dependence and non-stationarity of imperfect ADIs. Therefore, the history of imperfect ADIs would need to be collected as well, and the state-dependent optimal order-up-to point would also be a function of this history.

The following theorem states some monotonicity results.

Theorem 2. The following properties hold.

(i) $f_{n}^{\prime}(x, k) \geqslant f_{n+1}^{\prime}(x, k)$ for $n=1,2, \ldots, N$, for all $x$, and $k \geqslant 0$,

(ii) $J_{n-1}^{\prime}(y, k) \geqslant J_{n}^{\prime}(y, k)$ for $n=2,3, \ldots, N$, for all $y$, and $k \geqslant 0$,

(iii) $y_{n-1}(k) \leqslant y_{n}(k)$ for $n=2,3, \ldots, N$, for all $k \geqslant 0$,

where $f^{\prime}$ and $J^{\prime}$ refer to the derivatives taken with respect to the first arguments of $f$ and $J$, respectively.

Proof. Proof is provided in Appendix B.

Theorem 2(iii) simply states that, in any period $n-1$, as the system has a number of possible demand states to occur in the next period, it may position itself at a lower inventory position in period $n-1$, in order to be able to correct its inventory level to a more desirable position in period $n$.

Note that each of the monotonicity results provided in Theorem 2 are valid when the ADI sizes of the two consecutive periods of concern are the same. In other words, the order-up-to point of period $n$ can be less than that of period $n-1$ when the size of ADI available at the beginning of period $n$ is less than that of period $n-1$.

An upper bound on order-up-to levels can be deduced directly from Theorem 2 as follows:

Corollary 1. Optimal order-up-to level of the last period in the planning horizon for a given ADI size, $k$, is an upper bound for the optimal order-up-to level of any period with the same $k$.

This level can be derived as follows. We need to have $J_{N}^{\prime}(y, k)=0$ for $y=y_{N}(k)$. Therefore, $J_{N}^{\prime}(y, k)=\mathscr{L}^{\prime}(y, k)-\alpha c=0$, and then $c+\alpha^{L}\left(-b+(b+h) G_{k}(y)\right)-\alpha c=0$. Consequently,

$$
y_{N}(k)=G_{k}^{-1}\left(\frac{b-c(1-\alpha) \alpha^{-L}}{b+h}\right) .
$$

If the demand during effective lead time is taken to be approximately normal with mean $E[W(k)]$ and variance $\operatorname{Var}[W(k)]$, as computed in Eqs. (5) and (6), respectively, then

$$
y_{N}(k)=E[W(k)]+\sqrt{\operatorname{Var}[W(k)]} \cdot \Phi^{-1}\left(\frac{b-c(1-\alpha) \alpha^{-L}}{b+h}\right) .
$$

Note that this level can be calculated for any $k$; hence it may serve as a parametric upper bound for any value of the ADI size, $k$, in any period.

The following theorem characterizes the behavior of the optimal order-up-to point as related to the size of ADI.

Theorem 3. The following properties hold for $n=1,2, \ldots, N$, and for all $k$ and $\eta \geqslant 0$.

(i) $f_{n}^{\prime}(x, k) \geqslant f_{n}^{\prime}(x, k+\eta)$ for all $x$,

(ii) $J_{n}^{\prime}(y, k) \geqslant J_{n}^{\prime}(y, k+\eta)$ for all $y$,

(iii) $y_{n}(k) \leqslant y_{n}(k+\eta)$. 
Proof. Proof is provided in Appendix C.

The relation stated Theorem 3(iii) is rather intuitive: the order-up-to point increases as the size of ADI increases. Another property of the optimal order-up-to point as related to the size of ADI is stated in the following theorem.

Theorem 4. The following properties hold for $n=1,2, \ldots, N$, for $\eta_{1} \geqslant 0, \eta_{2} \geqslant 0$ such that $\eta_{1}+\eta_{2} \leqslant \eta$, and for all $k$.

(i) $J_{n}^{\prime}(y-\eta, k) \leqslant J_{n}^{\prime}\left(y-\eta_{1}, k+\eta_{2}\right)$ for all $y$,

(ii) $y_{n}(k)+\eta \geqslant y_{n}\left(k+\eta_{2}\right)+\eta_{1}$.

Proof. Proof is provided in Appendix D.

Theorem 4(ii) states that the marginal increase of the optimal order-up-to point cannot be greater than the marginal increase of information size that generates it. In specific, an additional unit of ADI will never cause the order-up-to level to increase more than one unit.

Note that efficient algorithms to compute optimal order-up-to levels can be devised by making use of the above properties. We provide one possible algorithm assuming discrete demand in Appendix E.

The form of the optimal policy and the qualitative results that we provide here are closely related to those that are obtained for submodular functions [12] and for other non-stationary demand models [24]. Although we are interested in finite horizon results, our results can be extended to infinite horizon, and a suitable policy iteration algorithm (see, for example, [13]) can be used (as an extension to the one provided in Appendix E) to obtain the state dependent inventory policy.

\section{Source segmentation}

In this section we discuss an extension to our model. We refer to the internet retailing example in our discussion for illustrative purposes, but we note that our results hold for the general problem, as long as it is possible to identify categorical differences between the distinct sources that generate imperfect ADI.

In the general internet customer framework, the least information that can be obtained by each connection to a product's website is the Internet Protocol (IP) address. The information about the number of visits and the previous orders given from that IP address alone can be evaluated to differentiate between those customers who tend to realize orders after providing an ADI and those who do not. The region or location of the connection may be of use, as well. For example, if the manufacturer supplies only the domestic market, then foreign connections can be disregarded.

There is also other information that can be gathered from potential customers, such as gender, age, profession, education, etc. While these pieces of information can be gathered through means like questionnaires, more reliable and practical information can be obtained via means like membership status, for which the customers provide information in the beginning. Upon availability of such information, ADI sources can be segmented accordingly, each having their respective customer reliability levels (that is, the probability of an ADI turning into a realized demand for each segment). A factorial design can be implemented to explore the main and interaction effects of the factors (such as age, education, etc.) on the reliability level, depending on the level of detail for such a segmentation. While it is possible to denote each factor separately on ADI, we consider $s=1,2, \ldots, S$ different segments, combining all levels of all factors. For example, if gender and five different age groups are of concern, then we have $S=10$ in our model, each $s$ standing for a different combination of the levels of these two factors.

The motivation behind segmentation, in case it is possible, is to make better use of imperfect ADI. A piece of information that belongs to a specific segment would otherwise be treated as information from any other segment. In case there are known differences between the reliability levels and/or information sojourn rates of segments, then segmentation can result in decreased system costs. 
We let each segment have a reliability level of $p^{s}$, and information sojourn rate of $r^{s}$, resulting in $u_{i}^{s}$, where

$$
u_{i}^{s}=p^{s} \sum_{j=0}^{\tau-i}\left(r^{s}\right)^{j}=p^{s}\left(1-\left(r^{s}\right)^{\tau-i+1}\right) /\left(1-\left(r^{s}\right)\right)
$$

for $i=1,2, \ldots, \tau$ and $s=1,2, \ldots, S$. Advance information on demand is collected separately for each segment, which we denote by $k^{s}$. Similarly, the random variable denoting the size of an (unobserved) ADI for each period for segment $s$ is $M^{s}$, with expectation $\mu_{M}^{s}$ and variance $\left(\sigma_{M}^{s}\right)^{2}$. Once ADI is collected for all segments, we have a vector $\mathbf{k}=\left(k^{1}, k^{2}, \ldots, k^{S}\right)$ constituting all the available ADI.

Let us consider the demand during effective lead time, that is, $W(\mathbf{k})$, at the beginning of period $n$. The arguments to be raised are similar to those in Section 2, hence will be skipped. If we assume independence between segments (and between each individual ADI, as before), then we can evaluate the expected value and the variance of $W^{s}\left(k^{s}\right)$, that is the demand originating from segment $s=1,2, \ldots, S$ during effective lead time, as

$$
\begin{aligned}
& E\left[W^{s}\left(k^{s}\right)\right]=k^{s} u_{1}^{s}+\mu_{M}^{s} \sum_{i=2}^{\tau} u_{i}^{s}, \\
& \operatorname{Var}\left[W^{s}\left(k^{s}\right)\right]=k^{s} u_{1}^{s}\left(1-u_{1}^{s}\right)+\sum_{i=2}^{\tau}\left\{\mu_{M}^{s} u_{i}^{s}\left(1-u_{i}^{s}\right)+\left(u_{i}^{s}\right)^{2}\left(\sigma_{M}^{s}\right)^{2}\right\},
\end{aligned}
$$

and consequently the expected value and the variance of the total demand during effective lead time as

$$
\begin{aligned}
& E[W(\mathbf{k})]=\sum_{s=1}^{S}\left[k^{s} u_{1}^{s}+\mu_{M}^{s} \sum_{i=2}^{\tau} u_{i}^{s}\right], \\
& \operatorname{Var}[W(\mathbf{k})]=\sum_{s=1}^{S}\left[k^{s} u_{1}^{s}\left(1-u_{1}^{s}\right)+\sum_{i=2}^{\tau}\left\{\mu_{M}^{s} u_{i}^{s}\left(1-u_{i}^{s}\right)+\left(u_{i}^{s}\right)^{2}\left(\sigma_{M}^{s}\right)^{2}\right\}\right] .
\end{aligned}
$$

The results that are obtained for the single source model can be adjusted to the source segmentation case by substituting the expected demand during effective lead time obtained in (19) and the variance of demand during effective lead time obtained in (20), when necessary. For example, the approximate upper bound derived in (18) turns out to be

$$
y_{N}(\mathbf{k})=E[W(\mathbf{k})]+\sqrt{\operatorname{Var}[W(\mathbf{k})]} \cdot \Phi^{-1}\left(\frac{b-c(1-\alpha) \alpha^{-L}}{b+h}\right) .
$$

We note that segmenting ADI sources and employing statistical tools for estimating corresponding reliability levels may impose difficulties if the amount of raw data to analyze is very large. In that case, it may be useful to apply an appropriate data mining technique.

\section{Value of information}

In this section our objective is to explore the value of information aspect of ADI, customer reliability level, and information sojourn rate. We first obtain explicit approximate expressions for the myopic (single decision epoch) problem in Section 5.1 and then we extend our analysis to the general (multi decision epoch) problem in Section 5.2.

\subsection{Value of information in the myopic problem}

Our exposition is based on the myopic (single decision epoch) problem. The myopic problem and its solution is presented in Appendix F. We first obtain the expected total relevant cost (TRC) term when ADI is utilized (ADI-case), and then compare it with the case where ADI is not utilized (NoADI-case). We make the comparison for $\alpha=1$.

The distribution of demand during the effective lead time is the convolution of a binomial distribution and $(\tau-1)$ distributions that depend on the distribution of $M$. For the analysis in this section, we apply normal approximation to both cases that are mentioned above. 
In order to make a meaningful comparison, the expected value and the variance of $M$ are assumed to be known. The analysis can be conducted either for the first period with $E[K]=\mu_{M}$, or assuming that the system is at stationarity so that $E[K]=\mu_{M} /(1-r)$. We assume a stationary system in what follows, which can easily be adapted to the case of the first period. Throughout this section we assume that $p>0$. If $p=0$, no matter what advance information there is on demand, the actual demand would be zero, and hence the value of information would trivially be zero.

\subsubsection{Advance demand information is utilized}

Here we consider the case in which the amount ordered, $y^{*}$, is based on the imperfect ADI size. Hence, we evaluate the expected cost term through conditioning on the ADI size, that is, $E\left[\operatorname{TRC}\left(y^{*}\right)\right]=E\left[E\left[\operatorname{TRC}\left(y^{*}\right) \mid k\right]\right]$. From (34),

$$
E\left[\operatorname{TRC}\left(y^{*}\right) \mid k\right]=(b+s) E\left[W(k)-y^{*}\right]^{+}+s\left(y^{*}-E[W(k)]\right)+y^{*} c .
$$

We note that the cost penalty due to the unsold items is accounted through a unit salvage cost, $s$, which is negative if salvage value exists. Here we replace $h$ in the multi-period model with $s$ to take into account the end-of-horizon effect. Making use of $E[W(k)]$ and $\operatorname{Var}[W(k)]$ terms derived in (7) and (8), and employing the normal approximation, we obtain

$$
\begin{aligned}
E\left[\operatorname{TRC}\left(y^{*}\right) \mid k\right]= & (b+s) \int_{y^{*}}^{\infty}\left(w-y^{*}\right) \mathrm{d} G_{k}(w)+s\left(y^{*}-\left(k u_{1}+\mu_{M} \sum_{i=2}^{\tau} u_{i}\right)\right)+y^{*} c \\
= & (b+s) \sqrt{k u_{1}\left(1-u_{1}\right)+\sum_{i=2}^{\tau}\left(\mu_{M} u_{i}\left(1-u_{i}\right)+u_{i}^{2} \sigma_{M}^{2}\right)} \\
& \cdot R_{u}\left(\frac{y^{*}-\left(k u_{1}+\mu_{M} \sum_{i=2}^{\tau} u_{i}\right)}{\sqrt{k u_{1}\left(1-u_{1}\right)+\sum_{i=2}^{\tau}\left(\mu_{M} u_{i}\left(1-u_{i}\right)+u_{i}^{2} \sigma_{M}^{2}\right)}}\right)+y^{*}(c+s)-s\left(k u_{1}+\mu_{M} \sum_{i=2}^{\tau} u_{i}\right),
\end{aligned}
$$

where

$$
R_{u}(r)=\int_{r}^{\infty}(t-r) \frac{1}{\sqrt{2 \pi}} \exp \left(-t^{2} / 2\right) \mathrm{d} t
$$

is the unit normal loss function.

Substituting $y^{*}$ derived in Appendix F (33), that is,

$$
y^{*}=k u_{1}+\mu_{M} \sum_{i=2}^{\tau} u_{i}+\sqrt{k u_{1}\left(1-u_{1}\right)+\sum_{i=2}^{\tau}\left(\mu_{M} u_{i}\left(1-u_{i}\right)+u_{i}^{2} \sigma_{M}^{2}\right)} \Phi^{-1}\left(\frac{b-c}{b+s}\right)
$$

into (22) yields

$$
\begin{aligned}
E\left[\operatorname{TRC}\left(y^{*}\right) \mid k\right]= & \sqrt{k u_{1}\left(1-u_{1}\right)+\sum_{i=2}^{\tau}\left(\mu_{M} u_{i}\left(1-u_{i}\right)+u_{i}^{2} \sigma_{M}^{2}\right)} \\
& \cdot\left[(b+s) R_{u}\left(\Phi^{-1}\left(\frac{b-c}{b+s}\right)\right)+(c+s) \Phi^{-1}\left(\frac{b-c}{b+s}\right)\right]+c\left(k u_{1}+\mu_{M} \sum_{i=2}^{\tau} u_{i}\right) .
\end{aligned}
$$

Let us define an auxiliary constant, $\beta$, for simplification

$$
\beta=\left[(b+s) R_{u}\left(\Phi^{-1}\left(\frac{b-c}{b+s}\right)\right)+(c+s) \Phi^{-1}\left(\frac{b-c}{b+s}\right)\right] .
$$


Also let

$$
\gamma=\sum_{i=2}^{\tau}\left(\mu_{M} u_{i}\left(1-u_{i}\right)+u_{i}^{2} \sigma_{M}^{2}\right)
$$

Then

$$
E\left[\operatorname{TRC}\left(y^{*}\right)\right]=\left(E\left[\sqrt{K u_{1}\left(1-u_{1}\right)+\gamma}\right]\right) \beta+c\left(E[K] u_{1}+\mu_{M} \sum_{i=2}^{\tau} u_{i}\right) .
$$

Let us refer to this term as $E\left[\mathrm{TRC}_{\mathrm{ADI}}\right]$.

\subsubsection{Advance demand information is not utilized}

In this case, the decision on how much to order is made without making use of the ADI size, $k$. The myopic problem results discussed in Appendix F still hold in general, except for the mean and the variance of the demand during effective lead time replacing $E[W(k)]$ and $\operatorname{Var}[W(k)]$ as follows:

$$
\begin{aligned}
& E[W]=E[K] u_{1}+\mu_{M} \sum_{i=2}^{\tau} u_{i}, \\
& \operatorname{Var}[W]=E[K] u_{1}\left(1-u_{1}\right)+\sum_{i=2}^{\tau}\left(\mu_{M} u_{i}\left(1-u_{i}\right)+u_{i}^{2} \sigma_{M}^{2}\right)+u_{1}^{2} \operatorname{Var}[K] \\
& =E[K] u_{1}\left(1-u_{1}\right)+\gamma+u_{1}^{2} \operatorname{Var}[K],
\end{aligned}
$$

where $E[K]$ and $\operatorname{Var}[K]$ are as expressed in (2) and (3), respectively. Following similar steps as in the ADI-case, and defining the order-up-to level in the NoADI-case as $\tilde{y}^{*}$, we obtain the expected total relevant costs as

$$
E\left[\operatorname{TRC}\left(\tilde{y}^{*}\right)\right]=\sqrt{\operatorname{Var}[W]} \beta+c E[W] .
$$

Substituting (24) and (25) in the above equation results in

$$
E\left[\operatorname{TRC}\left(\tilde{y}^{*}\right)\right]=\left(\sqrt{E[K] u_{1}\left(1-u_{1}\right)+\gamma+u_{1}^{2} \operatorname{Var}[K]}\right) \beta+c\left(E[K] u_{1}+\mu_{M} \sum_{i=2}^{\tau} u_{i}\right) .
$$

Let us refer to this term as $E\left[\mathrm{TRC}_{\mathrm{NO}-\mathrm{ADI}}\right]$.

The difference between these two expected cost terms, which is the reduction in expected relevant costs obtained by employing ADI, is the value of imperfect ADI for the myopic problem. Let us refer to this difference as $\Delta$, that is,

$$
\Delta=E\left[\mathrm{TRC}_{\mathrm{NO}-\mathrm{ADI}}\right]-E\left[\mathrm{TRC}_{\mathrm{ADI}}\right] .
$$

Then,

$$
\Delta=\left(\sqrt{E[K] u_{1}\left(1-u_{1}\right)+\gamma+u_{1}^{2} \operatorname{Var}[K]}-E\left[\sqrt{K u_{1}\left(1-u_{1}\right)+\gamma}\right]\right) \beta .
$$

We show in Appendix $\mathrm{G}$ that $\Delta>0$; hence, $E\left[\mathrm{TRC}_{\mathrm{NO}-\mathrm{ADI}}\right]>E\left[\mathrm{TRC}_{\mathrm{ADI}}\right]$ and consequently there is a positive value of ADI.

In the special case of $r=0,(27)$ simplifies into

$$
\Delta=\left(\sqrt{\left(\tau\left[p^{2} \sigma_{M}^{2}+p(1-p) \mu_{M}\right]\right)}-E\left[\sqrt{M p(1-p)+(\tau-1) \cdot\left[p^{2} \sigma_{M}^{2}+p(1-p) \mu_{M}\right]}\right]\right) \beta .
$$

Note that, if $p=0$, this would result in $\Delta=0$ for any $r$, as expected. If $\sigma_{M}^{2}=0$ and $r=0$, then $\sqrt{\mu_{M}}=E \sqrt{M}$, and consequently $\Delta=0$. This is because there is no uncertainty about $M$ since $\sigma_{M}^{2}=0$ and therefore there is no uncertainty about $K$, since $r=0$. However, if $r>0$, then $\Delta>0$ even for $\sigma_{M}^{2}=0$, since $\operatorname{Var}[K]>0$. That is, the stochasticity involved in the information carried from previous periods makes imperfect ADI still valuable even if the information arriving each period is deterministic. This result would not hold in the first period, since no information is carried from past then. In the other extreme, if $p=1$ then the advance demand becomes actual demand; that is, the advance information on demand is indeed perfect. In this case $r=0$ since 
$p+r \leqslant 1$. Consequently, $\Delta=\sigma_{M}(\sqrt{\tau}-\sqrt{\tau-1}) \beta$. Hence, the more variance $M$ has, the more the value of advance demand information attains, for any fixed effective lead time and a set of cost parameters. The intuition behind this result is clear: Increased uncertainty makes information more valuable. Note that the variance of total demand over the effective lead time and $\sigma_{M}^{2}$ have identical variability structures. In other words, the above discussion holds for the variance over the effective lead time demand as well; that is, the larger variance associated with the demand, the larger the value of ADI attains.

Another result that arises from the examination of (27) is that, when $r=0$ the value of imperfect ADI decreases as the effective lead time $(\tau)$ increases. The reason for this is the decreasing contribution (in proportion) of ADI in effective lead time demand as $\tau$ increases, since ADI has impact on a single period when $r=0$. In other words, the value of imperfect ADI increases as the proportion of effective lead time on which ADI is available increases.

While $\Delta$ is an important measure to test the sensitivity of the impact of ADI with respect to changes in the parameters, we define another measure to explore the relative sensitivity of ADI:

$$
\Delta_{\mathrm{f}}=\frac{\Delta}{E\left[\mathrm{TRC}_{\mathrm{ADI}}\right]}=\frac{E\left[\mathrm{TRC}_{\mathrm{NO}_{-} \mathrm{ADI}}\right]-E\left[\mathrm{TRC}_{\mathrm{ADI}}\right]}{E\left[\mathrm{TRC}_{\mathrm{ADI}}\right]},
$$

$\Delta_{\mathrm{f}}$ is the fractional penalty of not utilizing ADI. We first consider the special case of $p=1$, that is, ADI is perfect. Then,

$$
\Delta_{\mathrm{f}}=\frac{(\sqrt{\tau}-\sqrt{(\tau-1)}) \beta}{(\sqrt{(\tau-1)}) \beta+\tau c\left(\frac{\mu_{M}}{\sigma_{M}}\right)} .
$$

Eq. (30) reveals that, for any fixed effective lead time and set of cost parameters, $\Delta_{\mathrm{f}}$ increases as the coefficient of variation for $M, \sigma_{M} / \mu_{M}$, increases. In specific, when $\tau=1$,

$$
\Delta_{\mathrm{f}}=\frac{\beta}{c}\left(\frac{\sigma_{M}}{\mu_{M}}\right)
$$

that is, the impact is linear with respect to the coefficient of variation.

Now let us consider the case of imperfect information, that is, $0<p<1$. We first state some analytical results on $\Delta_{\mathrm{f}}$ when $r=0$ and $c=0$, and then present our experimental findings.

Proposition 1. The following properties hold for the fractional penalty of not utilizing ADI, $\Delta_{\mathrm{f}}$, when $r=0$ and $c=0$.

(i) For any given positive $\mu_{M}$ and $\sigma_{M}^{2}, \Delta_{\mathrm{f}}$ is an increasing function of $p$.

(ii) For any given positive $\mu_{M}$ and $0<p<1, \Delta_{\mathrm{f}}$ is an increasing function of $\sigma_{M}^{2}$.

Proof. Proof is provided in Appendix H.

In the empirical tests we conducted, we verified that Proposition 1 holds for the case of positive $r$ and $c$ as well.

Figs. 3 and 4 depict the percent penalty of not utilizing imperfect ADI, that is, $100 \Delta_{\mathrm{f}}$ versus $p$ for different levels of $r$ when $\tau=2$ and $\tau=5$, respectively. These figures are the results of the empirical tests in which $E\left[\mathrm{TRC}_{\mathrm{ADI}}\right]$ is computed by Monte Carlo simulation, and $E\left[\mathrm{TRC}_{\mathrm{NO}-\mathrm{ADI}}\right]$ and $\Delta_{\mathrm{f}}$ are calculated using Eqs. (26) and (29), respectively, where $\mu_{M}=200, \sigma_{M}^{2}=50^{2}$, the cost parameters being $b=10, s=2, c=1$, for $p \in(0.1, \ldots, 0.9)$ and $r \in(0.1, \ldots, 0.4) . E\left[\mathrm{TRC}_{\mathrm{ADI}}\right]$ is computed as follows:

- An ADI size, $k$, is generated from a normal distribution with parameters $E[K]$ and $\operatorname{Var}[K]$, a possible negative realization being truncated to zero. $E[K]$ and $\operatorname{Var}[K]$ are calculated by using Eqs. (2) and (3), where $\mu_{M}$ and $\sigma_{M}^{2}$ are as above.

- Using Eq. (23), $E\left[\operatorname{TRC}\left(y^{*} \mid k\right)\right]$ is calculated, the cost parameters being the same. 


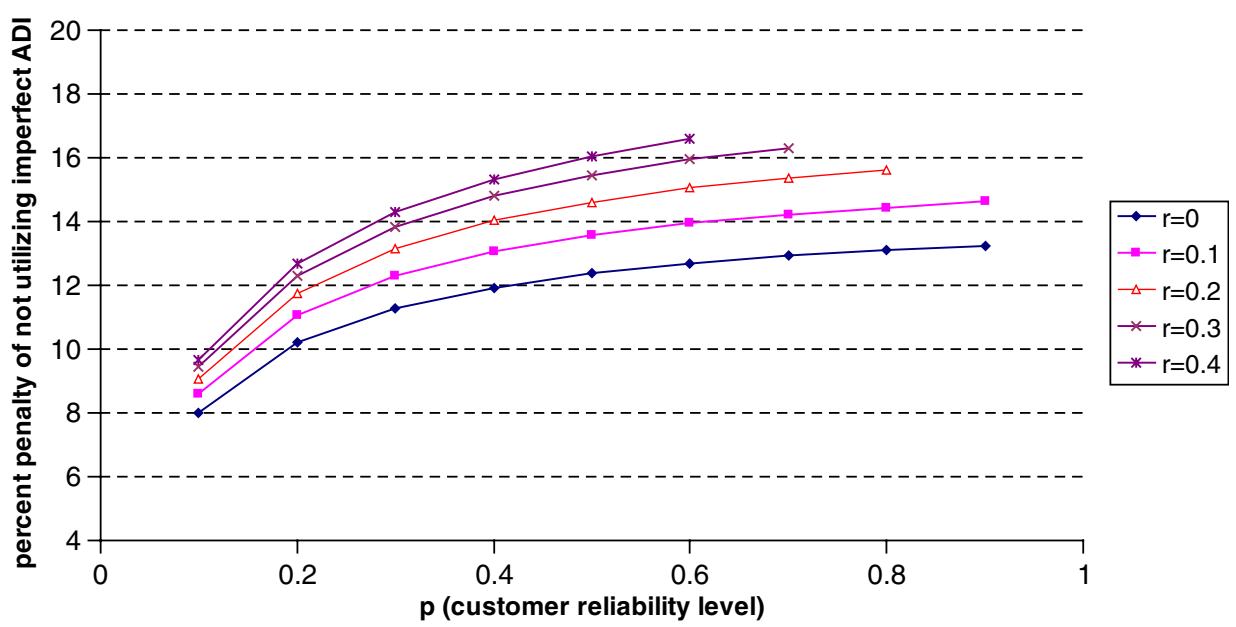

Fig. 3. Percent penalty of not utilizing imperfect ADI versus $p$ for $\tau=2$.

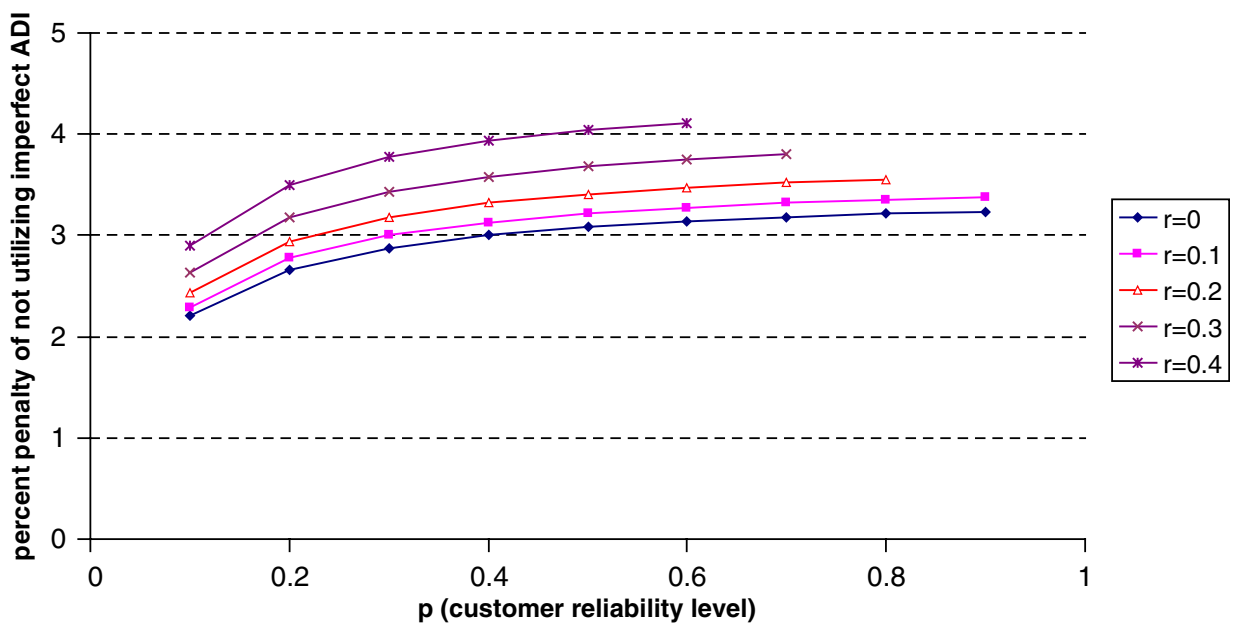

Fig. 4. Percent penalty of not utilizing imperfect ADI versus $p$ for $\tau=5$.

The average of 10,000 such realizations is taken as $E\left[\mathrm{TRC}_{\mathrm{ADI}}\right]$.

Fig. 5 depicts the percent penalty of not utilizing imperfect ADI versus the coefficient of variation of $M$ for different levels of $\tau$. In Fig. 5 we fix $p=0.3, r=0.2, \mu_{M}=200$ and vary $\tau$ in our Monte Carlo simulation.

The results that can be deduced from the empirical tests are in accordance with the analytical findings we had: The percent penalty of not utilizing imperfect ADI increases as $p$ increases and the coefficient of variation increases, the rest of the parameters being fixed. In other words, imperfect ADI becomes most beneficial under

- increased customer reliability level, hence decreased level of imperfectness of ADI, and

- increased variability in ADI sizes, hence increased variability in demand.

Figs. 3-5 also exhibit that $\Delta_{\mathrm{f}}$ decreases as $\tau$ increases, since the impact of ADI diminishes as the effective lead time increases, for a wide range of $p$ and $r$. Nevertheless, we note that an increase in $\tau$ does not necessarily result in decreased $\Delta_{\mathrm{f}}$. As a matter of fact, we observed that for very low $p$ and very high $r$ values, it may turn out that $\Delta_{\mathrm{f}}$ is higher for $\tau=2$ and $\tau=5$ than that with $\tau=1$. This is because the high value of $r$ makes the very unreliable information relatively more valuable for an effective lead time more than one period, since the 


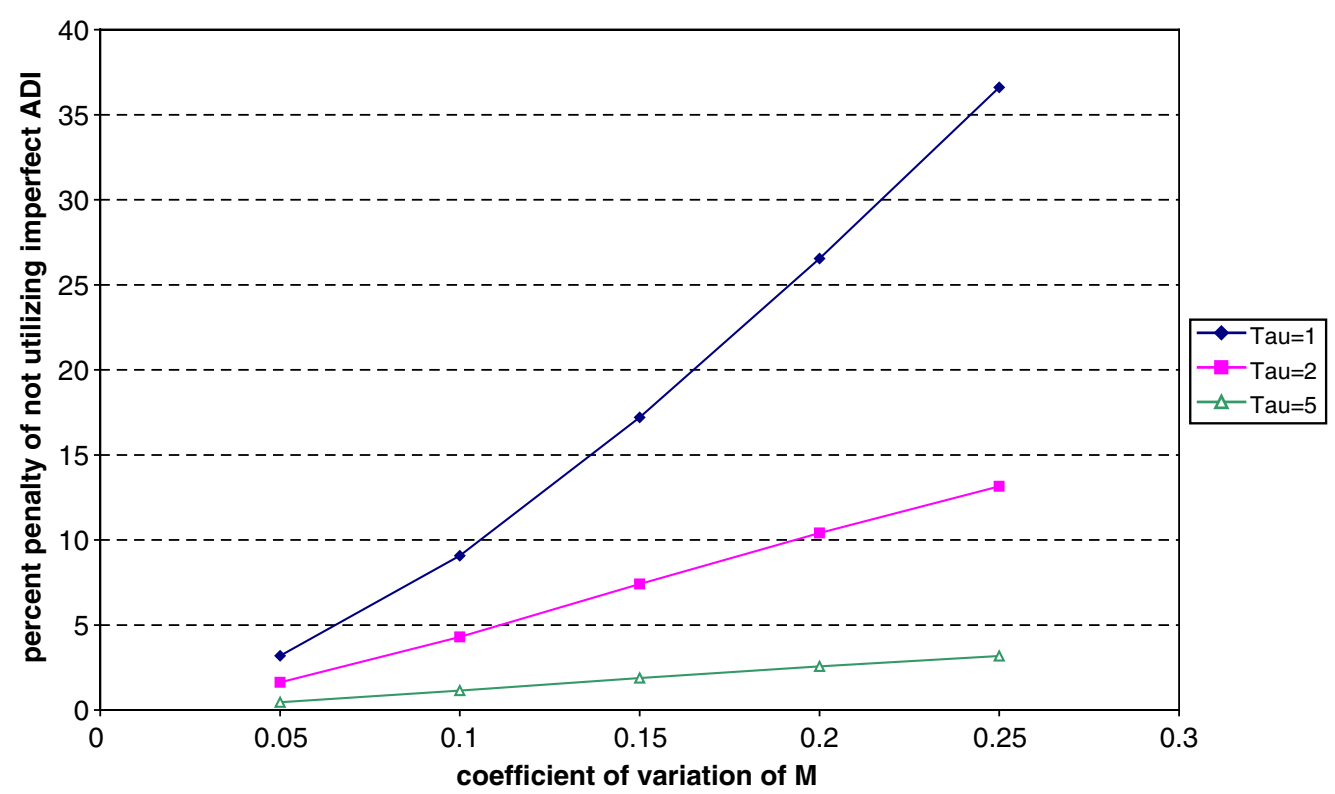

Fig. 5. Percent penalty of not utilizing imperfect ADI versus coefficient of variation of $M$.

"direct" effect of $r$ on the demand during the effective lead time (that is, other than changing the mean and variance of $K$ ) is seen only from the second period on of the effective lead time. However, as $p$ increases and $r$ decreases from the "extreme" values, $\Delta_{\mathrm{f}}$ increases as $\tau$ decreases, because the information decreases the uncertainty of demand during effective lead time in an increasing proportion as $\tau$ decreases, due to the dominating effect of the customer reliability level.

The effect of information sojourn rate on the fractional penalty of not utilizing imperfect ADI is more complicated and it may change both quantitatively and qualitatively for different parameter settings. Figs. 3 and 4 demonstrate that $\Delta_{\mathrm{f}}$ increases as $r$ increases for a given $p$, that is the value of information increases as the sojourn rate of the information increases for a fixed customer reliability level. This is due to the increased likelihood of the information being materialized as demand during the effective lead time for $\tau>1$. However, this result does not necessarily hold for all parameter settings, especially when $\tau=1$. In line with the discussion above, $r$ does not have an effect on the demand during the effective lead time for a given $k$ when $\tau=1$. Nevertheless, it increases the mean and the variance of $K$. It turns out that $\Delta_{\mathrm{f}}$ actually decreases as $r$ increases for a given $p$ when $\tau=1$. We observe this only for $\Delta_{\mathrm{f}}$, and we observe some non-monotonic behavior in the value of information ( $\Delta)$ as $r$ increases. The decrease in $\Delta_{\mathrm{f}}$ as $r$ increases can be seen also by an approximate analysis of $\Delta_{\mathrm{f}}$ for the special case of $c=0$ and $\mu_{M}=\sigma_{M}^{2}$. Using Taylor's approximation for $\sqrt{K}$ around $E[K]$ for the first three terms and taking expectations, we obtain

$$
E[\sqrt{K}] \approx \sqrt{E[K]}-\frac{1}{8}(E[K])^{-3 / 2} \operatorname{Var}[K] .
$$

Substituting $E[K]$ and $\operatorname{Var}[K]$ from (2) and (3), respectively, results in

$$
\Delta_{\mathrm{f}} \approx \frac{8 \mu_{M}}{\left(8 \mu_{M}-1+r\right) \sqrt{1-p}}-1,
$$

which is a term that decreases in $r$.

\subsection{Value of information in the general problem}

We solve the $N$-period problem following our dynamic model presented in Section 2.2, the properties of the optimal policy presented in Section 3, and the algorithm presented in Appendix E. We assume Poisson 
Table 2

Expected minimum costs in the ADI-case when $\tau=5$

\begin{tabular}{lrrrrrrr}
\hline & $r=0$ & $r=0.1$ & $r=0.2$ & $r=0.3$ & $r=0.4$ & $r=0.5$ & $r=0.6$ \\
\hline$p=0.1$ & 37.97 & 40.17 & 42.73 & 45.82 & 49.65 & 54.56 & 79.77 \\
$p=0.2$ & 55.52 & 58.74 & 62.56 & 67.12 & 72.72 & 89.22 \\
$p=0.3$ & 69.79 & 73.89 & 78.70 & 84.38 & 91.27 & 99.71 & 110.53 \\
$p=0.4$ & 82.37 & 87.22 & 92.81 & 99.43 & 107.25 & 116.62 & 127.97 \\
$p=0.5$ & 93.85 & 99.31 & 105.64 & 112.97 & 121.50 & 131.40 & \\
$p=0.6$ & 104.47 & 110.52 & 117.47 & 125.41 & 134.33 & & \\
$p=0.7$ & 114.48 & 121.06 & 128.52 & 137.09 & & & \\
$p=0.8$ & 123.98 & 131.02 & 139.01 & & & & \\
$p=0.9$ & 133.04 & 140.44 & & & & &
\end{tabular}

distribution for $M$. As in Section 5.1, the distribution of $M$ is assumed to be known both in the ADI-case and in the NoADI-case, which results in the distribution of the demand being the same in both cases, in order to make a meaningful comparison. We consider the system at stationarity. Consequently, the distribution of $K$ is Poisson with mean $E[K]=\mu_{M} /(1-r)$. The distribution of the demand during the effective lead time in the ADI-case, $W(k)$, is the convolution of $\operatorname{Binomial}\left(k, u_{1}\right)$ and Poisson $\left(\mu_{M} \sum_{i=2}^{\tau} u_{i}\right)$. The distribution of the demand during effective lead time observed by the decision maker in the NoADI-case is Poisson with mean $E[K] u_{1}+\mu_{M} \sum_{i=2}^{\tau} u_{i}$. We let $N=5, \mu_{M}=10, b=10, h=2, c=1$, and $\alpha=0.99$, for $p \in(0.1, \ldots, 1)$ and $r \in(0.1, \ldots, 0.6)$. Table 2 exhibits the expected total costs of the system in the ADI-case when $\tau=5$. We also demonstrate the optimal order-up-to levels at the beginning of period 1 when $p=0.3, r=0.2$, and $\tau=2$ in Fig. 6. Notice that the order-up-to level may stay the same even though the size of the available ADI increases. We note that the optimal order-up-to level of the NoADI-case under the same parameters is 10 .

Figs. 7 and 8 exhibit $100 \Delta_{\mathrm{f}}$ versus $p$ for different levels of $r$ when $\tau=2$ and $\tau=5$, respectively. These figures confirm the general findings that are discussed in Section 5.1, hence will not be repeated here. Also in line with the previous discussions, the value of information does not necessarily increase as $r$ increases for a given $p$. This is observed for $\tau=1$ (not displayed here) and for low values of $p$ when $\tau=2$. We note that precision

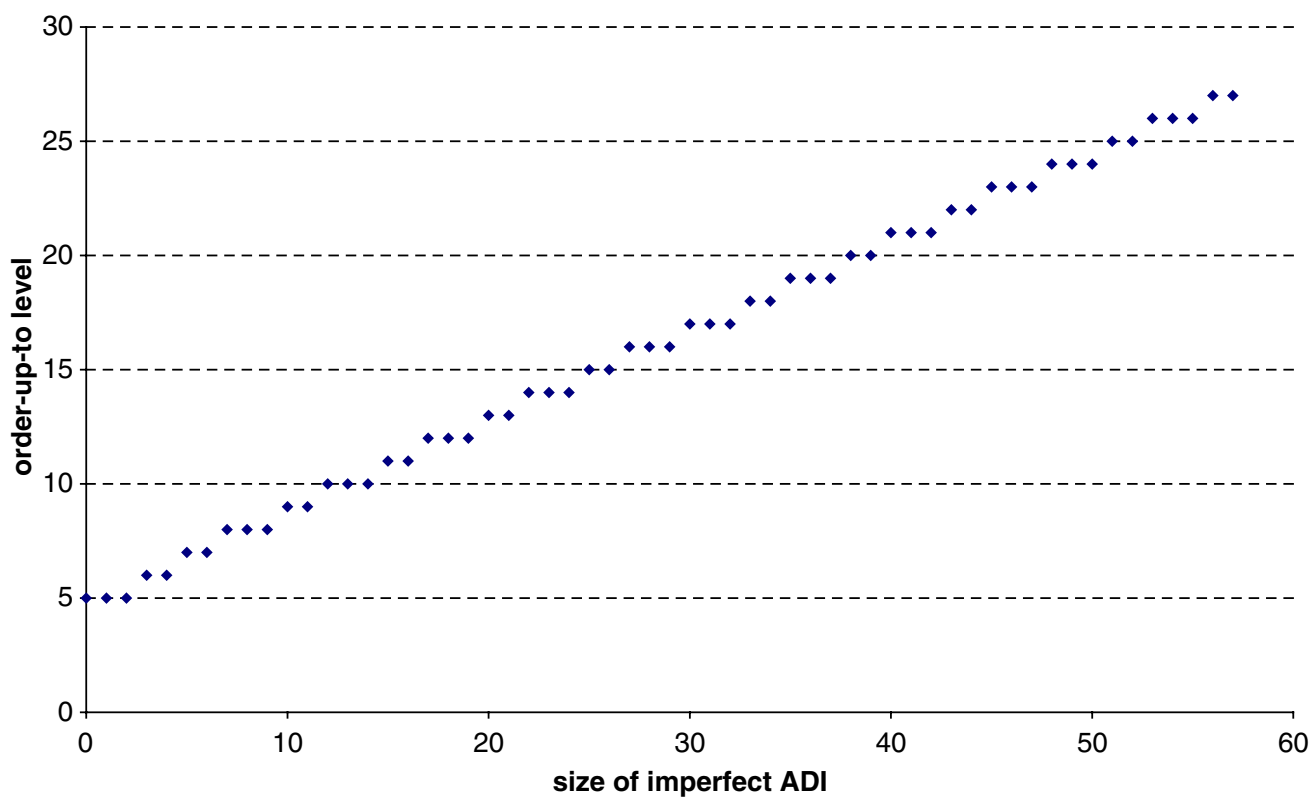

Fig. 6. Optimal order-up-to levels for $p=0.3, r=0.2, \tau=2$, and $N=5$. 


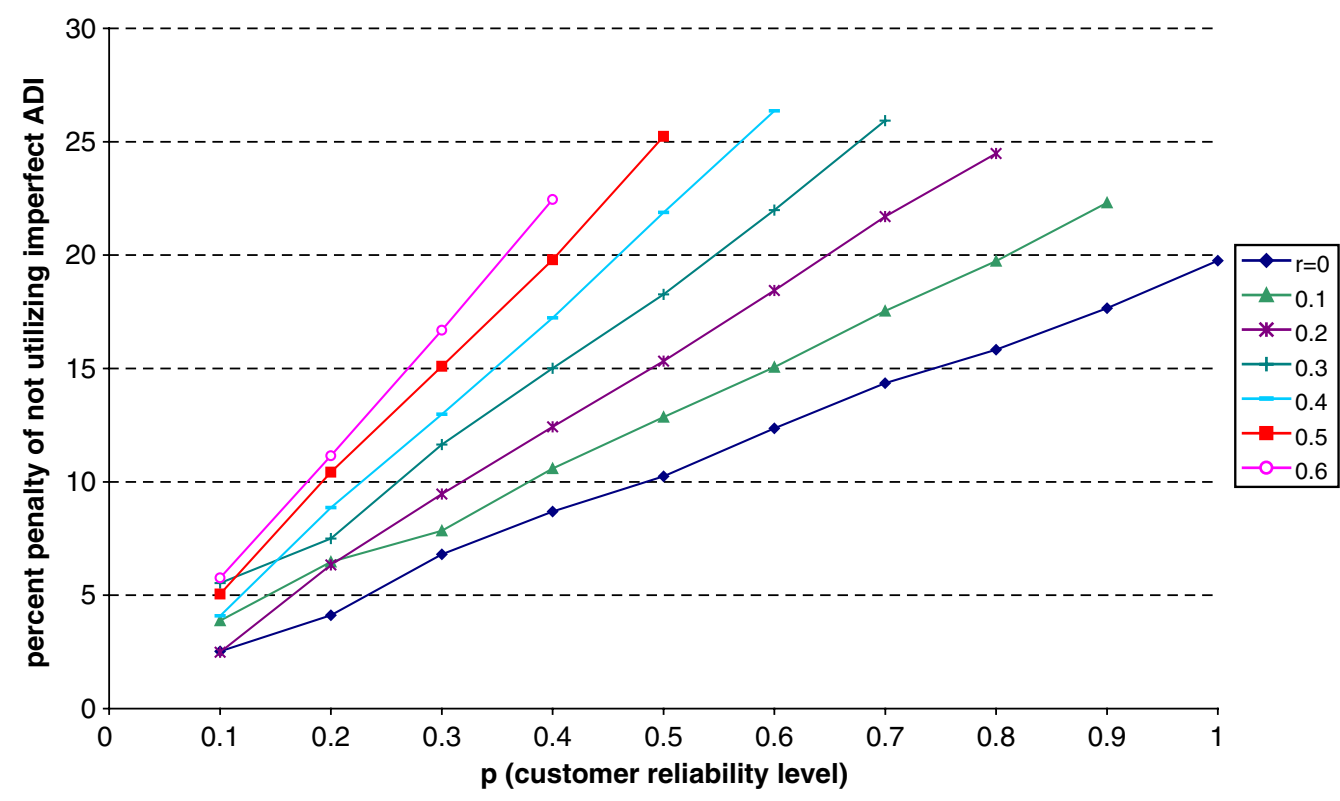

Fig. 7. Percent penalty of not utilizing imperfect ADI versus $p$ for $\tau=2$ and $N=5$.

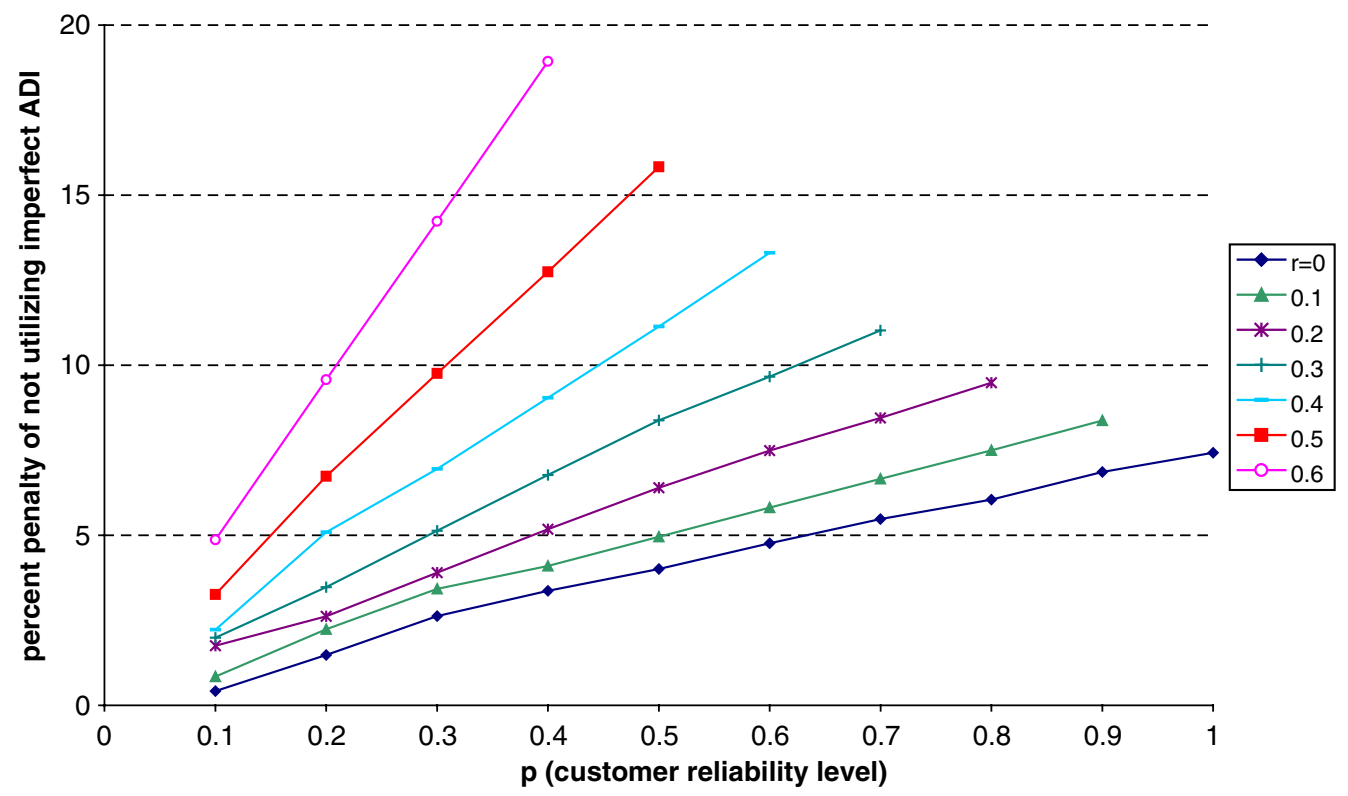

Fig. 8. Percent penalty of not utilizing imperfect ADI versus $p$ for $\tau=5$ and $N=5$.

difficulties and the assumption of discrete demand may add on top of the complex interactions to result in a non-monotonic behavior in $r$, especially when $\tau, p$, and $\mu_{M}$ are small.

\section{Conclusions and future research}

In this paper we have developed a model that incorporates imperfect ADI with inventory policies. We presented a fairly general probability structure for modelling imperfect advance demand information. Under our 
system settings, we have shown that the optimal ordering policy is of state-dependent order-up-to type, where the optimal order level is an increasing function of the ADI size. The optimal order-up-to levels are shown to be non-decreasing in time, for a given ADI size. Employing this idea, we generated an upper bound for the order-up-to level of any period (which is tight for the last period), depending on the ADI size. We have obtained some other useful structural properties of the optimal policy. Making use of these properties and assuming discrete demand, we provided an algorithm for computing the optimal order-up-to levels. We also outlined a natural way of extending the model under the availability of different segments of information sources.

Another contribution of this work is the derivation of the value of ADI for the myopic problem. Although the expression that is developed is valid for the myopic problem, it gives us clear ideas about the value of ADI in general. Combined with the analytical findings, the empirical tests we conducted both for the myopic problem and for the general problem demonstrate that imperfect ADI becomes most beneficial under decreased level of imperfectness of ADI and increased variability in demand. Our tests also demonstrated that while imperfect ADI is in general more valuable for increased information sojourn rate and shorter effective lead time, this does not necessarily hold for all possible values of the problem parameters.

An important extension to this study would be updating customer reliability parameters, $p$ and $r$, in time. We currently assume them to be fixed; however, either due to incorrect estimation or time dependence, $p$ and $r$ may need to be updated. In the business-to-business environments such as VMI and CPFR that are mentioned in the introduction, the updates are needed until a desired level of maturity in the partnership develops. This updating scheme can be performed in various ways. A possible way is to use Bayesian updates. In related literature this method is used by some authors in order to forecast the demand distribution more accurately in inventory models with unknown demand. See, for example, Azoury [1]. Other possible updating schemes may be time-series models as in Lovejoy [16], forecast evolution methods as in Güllü [9], or developing new analytical models to incorporate the information flow as in Gavirneni et al. [8].

Incorporation of "regular" demand (which does not provide advance information) into the structure handled in this study is also possible. If the regular demand is of equal priority with the other stream of demand on which imperfect ADI is collectible, then it is rather straightforward to extend the analysis in this study as long as the distribution of the total demand during effective lead time can be correctly assessed. Nevertheless, the problem becomes more interesting if these two demand classes have different priorities. Tan et al. [20] show that dynamic rationing policies as a function of imperfect ADI size need to be applied in that case; that is, some lower-priority demand might deliberately be backlogged or lost while carrying inventory, with an expectation of future demand from higher-priority customer class, based on the imperfect ADI.

Further interesting research could be the "configured demand" case in a multi-item environment, that is, the customers providing ADI on some configuration of the commodities. Consider the case of PC sales, for example. A customer might provide ADI on a specific setting of a PC (e.g. certain memory, hard-drive, monitor, CD-rom drive, etc.). This ADI could be considered as an ADI on each of the components in its bill of materials, some — or all — of which can be used to satisfy demands for some other PC settings, in case this ADI is not materialized. Then, a special postponement strategy as a function of ADI on components could be developed, resulting in reduced effective lead times and relevant costs.

\section{Acknowledgements}

The authors thank the anonymous referees for their suggestions which led to considerable improvement on the contents and the presentation of the article. Tarkan Tan was at Middle East Technical University and Atilim University, Refik Güllü was at Middle East Technical University, and Nesim Erkip was at Middle East Technical University and Technische Universiteit Eindhoven when parts of this research were carried out.

\section{Appendix A. Proof of Theorem 1}

For the proof we use induction. We first show that Theorem 1 holds for $n=N$ :

$$
J_{N}(y, k)=\mathscr{L}(y, k)+\alpha E\left[f_{N+1}\left(y-D(k), K_{N+1}(k)\right)\right]=\mathscr{L}(y, k)+\alpha E[-c(y-D(k))]=\mathscr{L}(y, k)+\alpha c(k p-y) .
$$


Part (i) follows since $\mathscr{L}(y, k)$ is convex in $y$ for all $k \geqslant 0$, and $\alpha c(k p-y)$ is linear-hence convex-in $y$. Part (iii) directly follows from convexity. As for (ii), we note that

$$
f_{N}(x, k)=-c x+ \begin{cases}J_{N}\left(y_{N}(k), k\right), & \text { if } x<y_{N}(k) \\ J_{N}(x, k), & \text { if } x \geqslant y_{N}(k)\end{cases}
$$

But then, because of convexity of $J_{N}$ and $-c x, f_{N}(x, k)$ is convex in $x$, which proves (ii).

Now let us assume that these results hold for period $n+1$, where $1 \leqslant n+1<N$. Our aim is to show that they hold for period $n$ as well. We first note that $f_{n+1}(x, k)$ is convex in $x$ for all values of $k$, due to the induction hypothesis. Now consider $E\left[f_{n+1}(x, k)\right]$. Since expectations can be written as the limits of Riemann-Stieltjes sums, and the positive-weighted sum of convex functions are convex (see, e.g. Heyman and Sobel [12]), then $E\left[f_{n+1}(x, k)\right]$ is convex too. Therefore, $J_{n}(y, k)=\mathscr{L}(y, k)+\alpha E\left[f_{n+1}\left(y-D(k), K_{n+1}(k)\right)\right]$ is convex, since $\mathscr{L}(y, k)$ is convex as well, which proves (i). Part (iii) directly follows from (i) again. And finally, writing $f_{n}$ as

$$
f_{n}(x, k)=-c x+ \begin{cases}J_{n}\left(y_{n}(k), k\right), & \text { if } x<y_{n}(k), \\ J_{n}(x, k), & \text { if } x \geqslant y_{n}(k)\end{cases}
$$

results in (ii), and this completes the proof.

\section{Appendix B. Proof of Theorem 2}

For the proof we use induction. Let us start with $n=N$. We have $f_{N+1}(x, k)=-c x, f_{N+1}^{\prime}(x, k)=-c$.

$$
\begin{aligned}
& f_{N}(x, k)=-c x+ \begin{cases}J_{N}\left(y_{N}(k), k\right), & \text { if } x \leqslant y_{N}(k), \\
J_{N}(x, k), & \text { if } x>y_{N}(k),\end{cases} \\
& f_{N}^{\prime}(x, k)=-c+ \begin{cases}0, & \text { if } x \leqslant y_{N}(k), \\
J_{N}^{\prime}(x, k), & \text { if } x>y_{N}(k) .\end{cases}
\end{aligned}
$$

But,

$$
\begin{aligned}
& J_{N}(y, k)=\mathscr{L}(y, k)+\alpha E\left[f_{N+1}\left(y-D(k), K_{N+1}(k)\right)\right]=\mathscr{L}(y, k)+\alpha c(k p-y), \\
& J_{N}^{\prime}(y, k)=\mathscr{L}^{\prime}(y, k)-\alpha c .
\end{aligned}
$$

So,

$$
f_{N}^{\prime}(x, k)=-c+ \begin{cases}0, & \text { if } x \leqslant y_{N}(k), \\ \mathscr{L}^{\prime}(x, k)-\alpha c, & \text { if } x>y_{N}(k) .\end{cases}
$$

Note that $\mathscr{L}^{\prime}(x, k)-\alpha c=J_{N}^{\prime}(x, k) \geqslant 0$ when $x>y_{N}(k)$. Consequently, $f_{N}^{\prime}(x, k) \geqslant f_{N+1}^{\prime}(x, k)$, as stated in (i).

We have $J_{N-1}^{\prime}(y, k)=\mathscr{L}^{\prime}(y, k)+\alpha E\left[f_{N}^{\prime}\left(y-D(k), K_{N}(k)\right)\right]$. But, as shown above, $f_{N}^{\prime}(x, k) \geqslant-c$ for all $x$ and $k$. Therefore, $E\left[f_{N}^{\prime}\left(y-D(k), K_{N}(k)\right)\right] \geqslant-c$, and hence, $J_{N-1}^{\prime}(y, k) \geqslant \mathscr{L}^{\prime}(y, k)-\alpha c=J_{N}^{\prime}(y, k)$. This proves (ii), which directly results in (iii).

Now let us assume that the induction hypotheses hold for period $n(2<n<N)$ as follows:

$$
\begin{aligned}
& f_{n}^{\prime}(x, k) \geqslant f_{n+1}^{\prime}(x, k), \\
& J_{n-1}^{\prime}(y, k) \geqslant J_{n}^{\prime}(y, k), \\
& y_{n-1}(k) \leqslant y_{n}(k) .
\end{aligned}
$$

As a result of the third item of this induction assumption, there can be three cases for the effective inventory position:

- $x \leqslant y_{n-1}(k) \leqslant y_{n}(k)$

In this case, $f_{n-1}^{\prime}(x, k)=-c=f_{n}^{\prime}(x, k)$.

- $y_{n-1}(k)<x \leqslant y_{n}(k)$

Here, we have $f_{n-1}^{\prime}(x, k)=-c+J_{n-1}^{\prime}(x, k)$, and $f_{n}^{\prime}(x, k)=-c$. But since $J_{n}^{\prime}(x, k)$ is nonnegative in this region, we obtain $f_{n-1}^{\prime}(x, k) \geqslant f_{n}^{\prime}(x, k)$. 
- $y_{n-1}(k) \leqslant y_{n}(k)<x$

Now, $f_{n-1}^{\prime}(x, k)=-c+J_{n-1}^{\prime}(x, k)$, and $f_{n}^{\prime}(x, k)=-c+J_{n}^{\prime}(x, k)$. But due to the induction assumption, $J_{n-1}^{\prime}(x, k) \geqslant J_{n}^{\prime}(x, k)$, and hence $f_{n-1}^{\prime}(x, k) \geqslant f_{n}^{\prime}(x, k)$.

Consequently, (i) holds. We also have $J_{n-2}^{\prime}(y, k)=\mathscr{L}^{\prime}(y, k)+\alpha E\left[f_{n-1}^{\prime}\left(y-D(k), K_{n-1}(k)\right)\right]$, and $J_{n-1}^{\prime}(y, k)=\mathscr{L}^{\prime}(y, k)+\alpha E\left[f_{n}^{\prime}\left(y-D(k), K_{n}(k)\right)\right]$. But, due to (i), $f_{n-1}^{\prime}\left(y-D(k), K_{n-1}(k)\right) \geqslant f_{n}^{\prime}\left(y-D(k), K_{n}(k)\right)$ for all possible values of $y-D(k)$ and $K_{n-1}(k)=K_{n}(k)$; and then, as discussed in the proof of Theorem 1 , $E\left[f_{n-1}^{\prime}\left(y-D(k), K_{n-1}(k)\right)\right] \geqslant E\left[f_{n}^{\prime}\left(y-D(k), K_{n}(k)\right)\right]$, which results in $J_{n-2}^{\prime}(y, k) \geqslant J_{n-1}^{\prime}(y, k)$. This proves (ii); and (iii) directly follows from (ii), which completes the proof.

\section{Appendix C. Proof of Theorem 3}

For the proof we use induction. We start with $n=N . f_{N+1}^{\prime}(x, k)=f_{N+1}^{\prime}(x, k+\eta)=-c$, which suffices for (i). As for (ii), we have $J_{N}^{\prime}(y, k)=\mathscr{L}^{\prime}(y, k)-\alpha c$, and $J_{N}^{\prime}(y, k+\eta)=\mathscr{L}^{\prime}(y, k+\eta)-\alpha c$. We note that $G_{k}(y) \geqslant G_{k+\eta}(y)$ for all $y$, because $X_{1}$ has Binomial distribution, and $X_{2}, X_{3}, \ldots X_{\tau}$ are independent of $k$ and $\eta$. Since $\mathscr{L}^{\prime}(y, k)=c+\alpha^{L}\left(-b+(h+b) G_{k}(y)\right)$ and $\mathscr{L}^{\prime}(y, k+\eta)=c+\alpha^{L}\left(-b+(h+b) G_{k+\eta}(y)\right)$, we result in $\mathscr{L}^{\prime}(y, k) \geqslant \mathscr{L}^{\prime}(y, k+\eta)$, and hence $J_{N}^{\prime}(y, k) \geqslant J_{N}^{\prime}(y, k+\eta)$, as desired. Part (iii) directly follows from (ii). Now let us assume that the induction hypotheses hold for period $n(2 \leqslant n<N)$ as follows:

$$
\begin{aligned}
& f_{n+1}^{\prime}(x, k) \geqslant f_{n+1}^{\prime}(x, k+\eta), \\
& J_{n}^{\prime}(y, k) \geqslant J_{n}^{\prime}(y, k+\eta), \\
& y_{n}(k) \leqslant y_{n}(k+\eta) .
\end{aligned}
$$

We note that

$$
f_{n}^{\prime}(x, k)=-c+ \begin{cases}0, & \text { if } x \leqslant y_{n}(k), \\ J_{n}^{\prime}(x, k), & \text { if } x>y_{n}(k)\end{cases}
$$

and

$$
f_{n}^{\prime}(x, k+\eta)=-c+ \begin{cases}0, & \text { if } x \leqslant y_{n}(k+\eta), \\ J_{n}^{\prime}(x, k+\eta), & \text { if } x>y_{n}(k+\eta) .\end{cases}
$$

From the third item of the induction assumption, there can be three cases for the effective inventory position:

- $x \leqslant y_{n}(k) \leqslant y_{n}(k+\eta)$

In this case, $f_{n}^{\prime}(x, k)=-c=f_{n}^{\prime}(x, k+\eta)$.

- $y_{n}(k)<x \leqslant y_{n}(k+\eta)$

Now we have $f_{n}^{\prime}(x, k)=-c+J_{n}^{\prime}(x, k)$, and $f_{n}^{\prime}(x, k+\eta)=-c$. But since $J_{n}^{\prime}(x, k)$ is nonnegative in this region, we obtain $f_{n}^{\prime}(x, k) \geqslant f_{n}^{\prime}(x, k+\eta)$.

- $y_{n}(k) \leqslant y_{n}(k+\eta)<x$

Here, we have $f_{n}^{\prime}(x, k)=-c+J_{n}^{\prime}(x, k)$, and $f_{n}^{\prime}(x, k+\eta)=-c+J_{n}^{\prime}(x, k+\eta)$. But due to the second induction assumption, $J_{n}^{\prime}(x, k) \geqslant J_{n}^{\prime}(x, k+\eta)$, and hence $f_{n}^{\prime}(x, k) \geqslant f_{n}^{\prime}(x, k+\eta)$.

Consequently, (i) holds. As for (ii), $J_{n-1}^{\prime}(y, k)=\mathscr{L}^{\prime}(y, k)+\alpha E\left[f_{n}^{\prime}\left(y-D(k), K_{n}(k)\right)\right]$, and $J_{n-1}^{\prime}(y, k+\eta)=$ $\mathscr{L}^{\prime}(y, k+\eta)+\alpha E\left[f_{n}^{\prime}\left(y-D(k+\eta), K_{n}(k+\eta)\right)\right], \quad$ so it suffices to show that $E\left[f_{n}^{\prime}\left(y-D(k), K_{n}(k)\right)\right] \geqslant$ $E\left[f_{n}^{\prime}\left(y-D(k+\eta), K_{n}(k+\eta)\right)\right]$, since it is already shown that $\mathscr{L}^{\prime}(y, k) \geqslant \mathscr{L}^{\prime}(y, k+\eta)$. We first note that $D(k+\eta)$ is stochastically larger (denoted $\geqslant_{\mathrm{st}}$ ) than $D(k)$, because $X_{1}$ has Binomial distribution. (The reader can refer to Ross [17] for a coverage of stochastic dominance relations.) Then, since $f_{n}(x, k)$ is convex in $x$ and therefore $f_{n}^{\prime}(x, k)$ is non-decreasing in $x$, we have $E\left[f_{n}^{\prime}\left(y-D(k), K_{n}(k)\right)\right] \geqslant E\left[f_{n}^{\prime}\left(y-D(k+\eta), K_{n}(k)\right)\right]$. Moreover, since $D(k)+A(k)$ has Binomial distribution (with parameters $k$ and $p+r) K_{n}(k+\eta) \geqslant_{\text {st }} K_{n}(k)$, which results in $E\left[f_{n}^{\prime}\left(y-D(k+\eta), K_{n}(k)\right)\right] \geqslant E\left[f_{n}^{\prime}\left(y-D(k+\eta), K_{n}(k+\eta)\right)\right]$ due to (i). Consequently, $E\left[f_{n}^{\prime}\left(y-D(k), K_{n}(k)\right)\right] \geqslant E\left[f_{n}^{\prime}\left(y-D(k+\eta), K_{n}(k+\eta)\right)\right]$ as desired. This proves (ii); and (iii) directly follows from (ii), which completes the proof. 


\section{Appendix D. Proof of Theorem 4}

For the proof we use induction. First $n=N$. Note that $J_{N}^{\prime}(y-\eta, k)=\mathscr{L}^{\prime}(y-\eta, k)-\alpha c$ and $J_{N}^{\prime}\left(y-\eta_{1}, k+\eta_{2}\right)=\mathscr{L}^{\prime}\left(y-\eta_{1}, k+\eta_{2}\right)-\alpha c$. Also,

$$
\begin{aligned}
& \mathscr{L}^{\prime}(y-\eta, k)=c+\alpha^{L}\left(-b+(h+b) G_{k}(y-\eta)\right), \\
& \mathscr{L}^{\prime}\left(y-\eta_{1}, k+\eta_{2}\right)=c+\alpha^{L}\left(-b+(h+b) G_{k+\eta_{2}}\left(y-\eta_{1}\right)\right) .
\end{aligned}
$$

Let $H_{1}(k)$ and $H_{1}\left(k+\eta_{2}\right)$ be two Binomial random variables with parameters $(k, p)$ and $\left(k+\eta_{2}, p\right)$, respectively. By our construction $H_{1}(k)+\eta \geqslant_{\text {st }} H_{1}\left(k+\eta_{2}\right)+\eta_{1}$. Therefore $G_{k}(y-\eta) \leqslant G_{k+\eta_{2}}\left(y-\eta_{1}\right)$ for all $y$, and hence $J_{N}^{\prime}(y-\eta, k) \leqslant J_{N}^{\prime}\left(y-\eta_{1}, k+\eta_{2}\right)$ for all $y$, which immediately gives $y_{N}(k)+\eta \geqslant y_{N}\left(k+\eta_{2}\right)+\eta_{1}$.

Suppose that the claim holds for $n+1$. Note that

$$
f_{n+1}^{\prime}(x-\eta, k)=-c+ \begin{cases}0, & \text { if } x-\eta \leqslant y_{n+1}(k), \\ J_{n+1}^{\prime}(x-\eta, k), & \text { if } x-\eta>y_{n+1}(k),\end{cases}
$$

and

$$
f_{n+1}^{\prime}\left(x-\eta_{1}, k+\eta_{2}\right)=-c+ \begin{cases}0, & \text { if } x-\eta_{1} \leqslant y_{n+1}\left(k+\eta_{2}\right), \\ J_{n+1}^{\prime}\left(x-\eta_{1}, k+\eta_{2}\right), & \text { if } x-\eta_{1}>y_{n+1}\left(k+\eta_{2}\right) .\end{cases}
$$

Now, for $x<y_{n+1}\left(k+\eta_{2}\right)+\eta_{1} \leqslant y_{n+1}(k)+\eta, f_{n+1}^{\prime}(x-\eta, k)=-c=f_{n+1}^{\prime}\left(x-\eta_{1}, k+\eta_{2}\right)$. For $y_{n+1}\left(k+\eta_{2}\right)+$ $\eta_{1} \leqslant x \leqslant y_{n+1}(k)+\eta, f_{n+1}^{\prime}(x-\eta, k)=-c$. On the other hand,

$$
f_{n+1}^{\prime}\left(x-\eta_{1}, k+\eta_{2}\right)=-c+J_{n+1}^{\prime}\left(x-\eta_{1}, k+\eta_{2}\right) \geqslant-c
$$

since in this region $x-\eta_{1} \geqslant y_{n+1}\left(k+\eta_{2}\right)$. For $x>y_{n+1}(k)+\eta$

$$
f_{n+1}^{\prime}\left(x-\eta_{1}, k+\eta_{2}\right)=-c+J_{n+1}^{\prime}\left(x-\eta_{1}, k+\eta_{2}\right) \geqslant-c+J_{n+1}^{\prime}(x-\eta, k)=f_{n+1}^{\prime}(x-\eta, k),
$$

by the induction hypothesis. Therefore, for all $x$ we have $f_{n+1}^{\prime}\left(x-\eta_{1}, k+\eta_{2}\right) \geqslant f_{n+1}^{\prime}(x-\eta, k)$.

Now we complete the induction. For all $y$ we have

$$
\begin{aligned}
& J_{n}^{\prime}(y-\eta, k)=\mathscr{L}^{\prime}(y-\eta, k)+\alpha E\left[f_{n+1}^{\prime}\left(y-\eta-D(k), K_{n+1}(k)\right)\right], \\
& J_{n}^{\prime}\left(y-\eta_{1}, k+\eta_{2}\right)=\mathscr{L}^{\prime}\left(y-\eta_{1}, k+\eta_{2}\right)+\alpha E\left[f_{n+1}^{\prime}\left(y-\eta_{1}-D\left(k+\eta_{2}\right), K_{n+1}\left(k+\eta_{2}\right)\right)\right] .
\end{aligned}
$$

First note that $D\left(k+\eta_{2}\right)=D(k)+D\left(\eta_{2}\right)$, where $D\left(\eta_{2}\right)$ is the additional (due to $\eta_{2}$ ) number of customers realized as demand in period $n$, and $D(k)$ and $D\left(\eta_{2}\right)$ are independent. Moreover, we can write $K_{n+1}\left(k+\eta_{2}\right)$ as $K_{n+1}\left(k+\eta_{2}\right)=K_{n+1}(k)+B\left(\eta_{2}\right)$ where $B\left(\eta_{2}\right)$ is the additional number of customers (due to $\left.\eta_{2}\right)$ who stay in the system for one more period. $K(k)$ and $B\left(\eta_{2}\right)$ are also independent. Last we observe that for any realization of these random variables $\eta_{1}+D\left(\eta_{2}\right)+B\left(\eta_{2}\right) \leqslant \eta$ since $D\left(\eta_{2}\right)+B\left(\eta_{2}\right) \leqslant \eta_{2}$. Therefore

$$
f_{n+1}^{\prime}\left(y-\eta-D(k), K_{n+1}(k)\right) \leqslant f_{n+1}^{\prime}\left(y-\eta_{1}-D\left(k+\eta_{2}\right), K_{n+1}\left(k+\eta_{2}\right)\right)
$$

and hence $J_{n}^{\prime}(y-\eta, k) \leqslant J_{n}^{\prime}\left(y-\eta_{1}, k+\eta_{2}\right)$, which immediately yields $y_{n}(k)+\eta \geqslant y_{n}\left(k+\eta_{2}\right)+\eta_{1}$. In particular, by letting $\eta_{1}=0$ and $\eta_{2}=\eta$ we obtain

$$
y_{n}(k)+\eta \geqslant y_{n}(k+\eta) \text {. }
$$

\section{Appendix E. An algorithm for computing optimal order-up-to points}

Do the following for $n=N$ to 1

1. Do the following for all possible values of $k$, starting from the smallest

(a) Calculate $\mathscr{F}(y, k)=E\left[f_{n+1}\left(y-D(k), K_{n+1}(k)\right]\right.$, for all possible values of $y$ (using $f_{N+1}(x, k)=-c x$ for $n=N)$. Here, expectation is taken over $D(k)$ and $K_{n+1}(k)$, using Eq. (1). Note that $D(k)$ and $K_{n+1}(k)$ are correlated.

(b) Find $y_{n}(k)$, which is the minimizer of $J_{n}(y, k)=\mathscr{L}(y, k)+\alpha \mathscr{F}(y, k)$, using 
i. convexity of $J_{n}$ (from Theorem 1(i)),

ii. $y_{n}(k) \leqslant y_{n+1}(k)$ (from Theorem 2(iii)),

iii. $y_{n}(k) \leqslant y_{n}(k+1)$ (from Theorem 3(iii)),

iv. $y_{n}(k+1) \leqslant y_{n}(k)+1$ (from Theorem $4($ ii) $)$,

where $\mathscr{L}(y, k)$ is defined in Eq. (17). Note that a full search for computing $y_{n}(k)$ is necessary only once (for the smallest possible $k$ value when $n=N$ ). Due to the properties stated above, the following suffices after that: For each increment of $k$ for a given $n$, it suffices to compare $y_{n}(k)$ with $y_{n}(k)+1$, and for each backwards increment of $n$ for the first possible value of $k$, the search for $y_{n}(k)$ is bounded up with $y_{n+1}(k)$.

2. Calculate $f_{n}(x, k)$ for all possible values of $x$ and $k$, using Eq. (15).

The computations above are performed for values of $x, y$, and $k$ within prespecified precision bounds.

\section{Appendix F. Myopic problem}

In the myopic problem, there is one decision epoch (the beginning of period 1) and we have the initial ADI of size $k$, on the demand to be realized during the effective lead time. In accordance with the assumptions stated in Section 2, the last demand is realized at the beginning of period $\tau+1$ and the costs that may be incurred before period $L+1$ are not considered. Whatever is left at the end of the planning horizon will be salvaged with a unit cost of $s$ (which is negative if salvage value exists), and unmet demand will be penalized with a unit cost of $b$. We also have a unit procurement (or production) cost, $c$. Under these settings, the problem is a special case of the newsboy problem. Hence, the optimal order-up-to point $y^{*}$ can be found as

$$
y^{*}=\inf \left\{y: \operatorname{Pr}\{W(k) \geqslant y\} \leqslant \frac{c+s}{b+s}\right\} .
$$

If the distribution of $W(k)$ is approximated by normal distribution, then

$$
y^{*}=E[W(k)]+\sqrt{\operatorname{Var}[W(k)]} \Phi^{-1}\left(\frac{b-c}{b+s}\right),
$$

with $E[W(k)]$ and $\operatorname{Var}[W(k)]$ are as derived in (7) and (8), respectively. Consequently,

$$
y^{*}=k u_{1}+\mu_{M} \sum_{i=2}^{\tau} u_{i}+\sqrt{k u_{1}\left(1-u_{1}\right)+\sum_{i=2}^{\tau}\left(\mu_{M} u_{i}\left(1-u_{i}\right)+u_{i}^{2} \sigma_{M}^{2}\right)} \Phi^{-1}\left(\frac{b-c}{b+s}\right) .
$$

The expected total relevant cost term, conditioned on the ADI size, can be evaluated as follows:

$$
\begin{aligned}
E[\operatorname{TRC} \mid k] & =b E[W(k)-y]^{+}+s E[y-W(k)]^{+}+y c \\
& =b E[W(k)-y]^{+}+s\left(E[y-W(k)]+E[W(k)-y]^{+}\right)+y c \\
& =(b+s) E[W(k)-y]^{+}+s(y-E[W(k)])+y c .
\end{aligned}
$$

\section{Appendix G. Proof of $\Delta>0$}

$$
\Delta=\left(\sqrt{E[K] u_{1}\left(1-u_{1}\right)+\gamma+u_{1}^{2} \operatorname{Var}[K]}-E\left[\sqrt{K u_{1}\left(1-u_{1}\right)+\gamma}\right]\right) \beta \text {. Let } \Delta_{1}=\left(\sqrt{E[K] u_{1}\left(1-u_{1}\right)+\gamma+u_{1}^{2} \operatorname{Var}[K]}\right.
$$
$\left.-E\left[\sqrt{K u_{1}\left(1-u_{1}\right)+\gamma}\right]\right)$, so that $\Delta=\Delta_{1} \beta$. From Jensen's inequality, it follows that $\left(\sqrt{E[K] u_{1}\left(1-u_{1}\right)+\gamma} \geqslant\right.$ $\left.E\left[\sqrt{K u_{1}\left(1-u_{1}\right)+\gamma}\right]\right)$, and since $u_{1}^{2} \operatorname{Var}[K]>0$ we obtain $\Delta_{1}>0$. We next show that $\beta>0$. This proof is based on a similar one by Güllü [10].

$$
\beta=(b+s) G_{u}\left(\Phi^{-1}\left(\frac{b-c}{b+s}\right)\right)+(c+s) \Phi^{-1}\left(\frac{b-c}{b+s}\right) .
$$


Let $\phi(r)$ denote the pdf of standard normal distribution. Then, since $\phi^{\prime}(r)=-r \phi(r)$, we have

$$
\int_{u}^{\infty} r \phi(r) \mathrm{d} r=\phi(u)
$$

Therefore, $G_{u}(z)=\phi(z)-z(1-\Phi(z)) \forall z$.

So,

$$
\begin{aligned}
G_{u}\left(\Phi^{-1}\left(\frac{b-c}{b+s}\right)\right) & =\phi\left(\Phi^{-1}\left(\frac{b-c}{b+s}\right)\right)-\Phi^{-1}\left(\frac{b-c}{b+s}\right)\left[1-\Phi\left(\Phi^{-1}\left(\frac{b-c}{b+s}\right)\right)\right] \\
& =\phi\left(\Phi^{-1}\left(\frac{b-c}{b+s}\right)\right)-\Phi^{-1}\left(\frac{b-c}{b+s}\right)\left[\frac{s+c}{b+s}\right] .
\end{aligned}
$$

Hence,

$$
\begin{aligned}
\beta & =(b+s)\left\{\phi\left(\Phi^{-1}\left(\frac{b-c}{b+s}\right)\right)-\Phi^{-1}\left(\frac{b-c}{b+s}\right)\left(\frac{s+c}{b+s}\right)\right\}+(s+c) \Phi^{-1}\left(\frac{b-c}{b+s}\right) \\
& =(b+s) \phi\left(\Phi^{-1}\left(\frac{b-c}{b+s}\right)\right)>0, \quad \text { and consequently, } \Delta>0 .
\end{aligned}
$$

\section{Appendix H. Proof of Proposition 1}

Proof of (i)

$$
\Delta_{\mathrm{f}}=\frac{\sqrt{\tau \gamma}}{E[\sqrt{M p(1-p)+(\tau-1) \gamma}]}-1,
$$

where $\gamma=p^{2} \sigma_{M}^{2}+p(1-p) \mu_{M}$. In order to show that $\Delta_{\mathrm{f}}$ increases as $p$ increases, it suffices to show that $\frac{\partial \Delta_{\mathrm{f}}}{\partial p}>0$ for all $0<p<1$. We consider the cases of $\tau=1$ and $\tau>1$ separately.

Case 1. $\tau=1$ :

$\Delta_{\mathrm{f}}=\frac{\sqrt{\gamma}}{E[\sqrt{M}] \sqrt{p(1-p)}}-1$. Then, we need to show that

$$
\frac{\partial \Delta_{\mathrm{f}}}{\partial p}=\frac{\frac{1}{2} \frac{\gamma^{\prime}}{\sqrt{\gamma}} E[\sqrt{M}] \sqrt{p(1-p)}-\frac{1}{2} \frac{(1-2 p)}{\sqrt{p(1-p)}} E[\sqrt{M}] \sqrt{\gamma}}{(E[\sqrt{M}] \sqrt{p(1-p)})^{2}}>0 .
$$

Let us assume that (35) does not hold. Then,

$$
\frac{1}{2} \frac{\gamma^{\prime}}{\sqrt{\gamma}} E[\sqrt{M}] \sqrt{p(1-p)} \leqslant \frac{1}{2} \frac{(1-2 p)}{\sqrt{p(1-p)}} E[\sqrt{M}] \sqrt{\gamma},
$$

since

$$
(E[\sqrt{M}] \sqrt{p(1-p)})^{2}>0 .
$$

Simplification yields $\gamma^{\prime} p(1-p) \leqslant \gamma(1-2 p)$. Substituting $\gamma$ and $\gamma^{\prime}$, we obtain

$$
\left(2 p \sigma_{M}^{2}+(1-2 p) \mu_{M}\right)(p(1-p)) \leqslant\left(p^{2} \sigma_{M}^{2}+p(1-p) \mu_{M}\right)(1-2 p) .
$$

Simplifying the above inequality results in $\sigma_{M}^{2} \leqslant 0$, which contradicts to $\sigma_{M}^{2}>0$. Hence, (35) must hold.

Case 2. $\tau>1$ :

We need to show that

$$
\frac{\partial \Delta_{\mathrm{f}}}{\partial p}=\frac{\frac{1}{2} \frac{\tau \gamma^{\prime}}{\sqrt{\tau \gamma}} E[\sqrt{M p(1-p)+(\tau-1) \gamma}]-E\left[\frac{1}{2} \frac{M(1-2 p)+(\tau-1) \gamma^{\prime}}{\sqrt{M p(1-p)+(\tau-1) \gamma}}\right] \sqrt{\tau \gamma}}{(E[\sqrt{M p(1-p)+(\tau-1) \gamma}])^{2}}>0
$$


which is equivalent to showing that

$$
E\left[\frac{1}{2} \frac{\tau \gamma^{\prime}}{\sqrt{\tau \gamma}} \sqrt{M p(1-p)+(\tau-1) \gamma}-\frac{1}{2} \frac{M(1-2 p)+(\tau-1) \gamma^{\prime}}{\sqrt{M p(1-p)+(\tau-1) \gamma}} \sqrt{\tau \gamma}\right]>0,
$$

because $(E[\sqrt{M p(1-p)+(\tau-1) \gamma}])^{2}>0$ and expectation is a linear operator. (Note that $\gamma$ and $\gamma^{\prime}$ are constant for a given set of $\mu_{M}, \sigma_{M}^{2}$, and $p$.)

In order to show (36), we will show that

$$
\frac{1}{2} \frac{\tau \gamma^{\prime}}{\sqrt{\tau \gamma}} \sqrt{M p(1-p)+(\tau-1) \gamma}-\frac{1}{2} \frac{M(1-2 p)+(\tau-1) \gamma^{\prime}}{\sqrt{M p(1-p)+(\tau-1) \gamma}} \sqrt{\tau \gamma}>0
$$

for all $M>0$, since the expectation term in (36) is a positive-weighted Riemann-Stieltjes sum of the term in (37) for all values of $M$. When $M=0$ (and remembering that $\tau>1$ ), $\frac{\partial \Delta_{\mathrm{f}}}{\partial p}$ equals zero, which is intuitive, because there would be no impact of a change in $p$ when ADI size is zero. But since $\sigma_{M}^{2}>0$, we have $\operatorname{Pr}\{M=0\}<1$, and the argument that "showing (37) for all $M>0$ is sufficient for showing (36)" holds.

For a given $M>0$, let us assume that (37) does not hold. Then,

$$
\frac{1}{2} \frac{\tau \gamma^{\prime}}{\sqrt{\tau \gamma}} \sqrt{M p(1-p)+(\tau-1) \gamma} \leqslant \frac{1}{2} \frac{M(1-2 p)+(\tau-1) \gamma^{\prime}}{\sqrt{M p(1-p)+(\tau-1) \gamma}} \sqrt{\tau \gamma} .
$$

Simplification yields $\gamma^{\prime} p(1-p) \leqslant \gamma(1-2 p)$ as in case 1 , resulting in $\sigma_{M}^{2} \leqslant 0$, which contradicts with $\sigma_{M}^{2}>0$. Hence, (37) must hold for all $M>0$, which completes the proof of (i).

Proof of (ii)

Similarly, we need to show that $\frac{\partial \Delta_{\mathrm{f}}}{\partial \sigma_{M}^{2}}>0$ for all $\sigma_{M}^{2}>0$. We follow the same arguments as in the proof of (i). For the case $\tau=1$,

$$
\frac{\partial \Delta_{\mathrm{f}}}{\partial \sigma_{M}^{2}}=\frac{\frac{1}{2} \frac{p^{2}}{\sqrt{\gamma}} E[\sqrt{M}] \sqrt{p(1-p)}}{(E[\sqrt{M}] \sqrt{p(1-p)})^{2}} .
$$

Assuming that (38) is less than or equal to zero results in $p^{2} \leqslant 0$, which contradicts to $p>0$. For the case $\tau>1$,

$$
\frac{\partial \Delta_{\mathrm{f}}}{\partial \sigma_{M}^{2}}=\frac{\frac{1}{2} \frac{\tau p^{2}}{\sqrt{\tau \gamma}} E[\sqrt{M p(1-p)+(\tau-1) \gamma}]-E\left[\frac{1}{2} \frac{(\tau-1) p^{2}}{\sqrt{M p(1-p)+(\tau-1) \gamma}}\right] \sqrt{\tau \gamma}}{(E[\sqrt{M p(1-p)+(\tau-1) \gamma}])^{2}} .
$$

It suffices to show that

$$
\frac{1}{2} \frac{\tau p^{2}}{\sqrt{\tau \gamma}} \sqrt{M p(1-p)+(\tau-1) \gamma}-\frac{1}{2} \frac{(\tau-1) p^{2}}{\sqrt{M p(1-p)+(\tau-1) \gamma}} \sqrt{\tau \gamma}>0
$$

for all $M>0$. Assuming that (39) does not hold results in $M p(1-p) \leqslant 0$, and this contradiction completes the proof.

\section{References}

[1] K.S. Azoury, Bayes solution to dynamic inventory models under unknown demand distribution, Management Science 31 (1985) $1150-1160$.

[2] F. Chen, J.-S. Song, Optimal policies for multiechelon inventory problems with Markov-modulated demand, Operations Research 49 (2001) 226-234.

[3] K.L. Cheung, A.X. Zhang, The impact of inventory information distortion due to customer order cancellations, Naval Research Logistics 46 (1999) 213-231.

[4] G.A. DeCroix, V.S. Mookerjee, Purchasing demand information in a stochastic-demand inventory, European Journal of Operational Research 102 (1997) 36-57.

[5] N.P. Dellaert, M.T. Melo, Approximate solutions for a stochastic lot-sizing problem with partial customer-order information, European Journal of Operational Research 150 (2003) 163-180.

[6] F.F. Easton, D.R. Moodie, Pricing and lead time decisions for make-to-order firms with contingent orders, European Journal of Operational Research 116 (1999) 305-318. 
[7] G. Gallego, Ö. Özer, Integrating replenishment decisions with advance demand information, Management Science 47 (2001) 1344 1360.

[8] S. Gavirneni, R. Kapuscinski, S. Tayur, Value of information in capacitated supply chains, Management Science 45 (1999) 16-24.

[9] R. Güllü, On the value of information in dynamic production/inventory problems under forecast evolution, Naval Research Logistics 43 (1996) 289-303.

[10] R. Güllü, A two-echelon allocation model and the value of information under correlated forecasts and demands, European Journal of Operational Research 99 (1997) 386-400.

[11] R. Hariharan, P. Zipkin, Customer-order information, leadtimes, and inventories, Management Science 41 (1995) $1599-1607$.

[12] D. Heyman, M. Sobel, Stochastic Models in Operations Research, vol. 2, Stochastic Optimization, Dover, Mineola, NY, 2004.

[13] R.A. Howard, Dynamic Programming and Markov Processes, MIT Press, Cambridge, 1960.

[14] F. Karaesmen, J.A. Buzacott, Y. Dallery, Integrating advance order information in make-to-stock production systems, IIE Transactions 34 (2002) 649-662.

[15] H.L. Lee, V. Padmanabhan, S. Whang, Information distortion in a supply chain: The bullwhip effect, Management Science 43 (1997) 546-558.

[16] W.S. Lovejoy, Myopic policies for some inventory models with uncertain demand distributions, Management Science 36 (1990) 724 738.

[17] S. Ross, Stochastic Processes, second ed., John Wiley \& Sons, New York, NY, 1996.

[18] S.P. Sethi, F. Cheng, Optimality of $(s, S)$ policies in inventory models with Markovian demand, Operations Research 45 (1997) $931-$ 939.

[19] J.-S. Song, P. Zipkin, Inventory control in a fluctuating demand environment, Operations Research 41 (1993) 351-370.

[20] T. Tan, R. Güllü, N. Erkip, Using imperfect advance demand information in ordering and rationing decisions, Working Paper WP130, Eindhoven University of Technology, Department of Technology Management, Eindhoven, The Netherlands, 2005.

[21] U.W. Thonemann, Improving supply-chain performance by sharing advance demand information, European Journal of Operational Research 142 (2002) 81-107.

[22] J.T. Treharne, C.R. Sox, Adaptive inventory control for non-stationary demand and partial information, Management Science 48 (2002) 607-624.

[23] K. Van Donselaar, L.R. Kopczak, M. Wouters, The use of advance demand information in a project-based supply chain, European Journal of Operations Research 130 (2001) 519-538.

[24] A.F. Veinott Jr., Optimal policy for a multi-product, dynamic nonstationary inventory problem, Management Science 12 (1965) 206222.

[25] K. Zhu, U.W. Thonemann, Modeling the benefits of sharing future demand information, Operations Research 52 (2003) $136-147$. 